\title{
Review Article \\ Recent Advances in Distributed Acoustic Sensing Based on Phase-Sensitive Optical Time Domain Reflectometry
}

\author{
Yonas Muanenda \\ Institute of Communication, Information and Perception Technologies (TeCIP), Scuola Superiore Sant'Anna, Via G. Moruzzi 1, \\ 56124 Pisa, Italy \\ Correspondence should be addressed to Yonas Muanenda; y.muanenda@sssup.it
}

Received 13 October 2017; Revised 28 March 2018; Accepted 5 April 2018; Published 13 May 2018

Academic Editor: Banshi D. Gupta

Copyright (c) 2018 Yonas Muanenda. This is an open access article distributed under the Creative Commons Attribution License, which permits unrestricted use, distribution, and reproduction in any medium, provided the original work is properly cited.

\begin{abstract}
Distributed acoustic sensing (DAS) using coherent Rayleigh backscattering in an optical fiber has become a ubiquitous technique for monitoring multiple dynamic events in real time. It has continued to constitute a steadily increasing share of the fiber-optic sensor market, thanks to its interesting applications in many safety, security, and integrity monitoring systems. In this contribution, an overview of the recent advances of research in DAS based on phase-sensitive optical time domain reflectometry ( $\phi$-OTDR) is provided. Some advanced techniques used to enhance the performance of $\phi$-OTDR sensors for measuring backscattering intensity changes through reduction of measurement noise are presented, in addition to methods used to increase the dynamic measurement capacity of $\phi$-OTDR schemes beyond conventional limits set by the sensing distance. Recent $\phi$-OTDR configurations which significantly enhance the measurement spatial resolution, including those which decouple it from the probing pulse width, are also discussed. Finally, a review of recent advances in more precise quantitative measurement of an external impact based on frequency shift and phase demodulation methods using simple direct detection $\phi$-OTDR schemes is given.
\end{abstract}

\section{Introduction}

Distributed fiber-optic sensors have become ubiquitous in the measurement of changes in environmental parameters induced by multiple events over long distances. They have interesting applications in a number of safety and integrity monitoring applications including, among others, transportation, oil and gas, and process control systems [1]. While some monitoring scenarios involve measurement of slowly changing parameters such as temperature or static strain, a number of natural phenomena that need to be monitored in many other scenarios involve fast changing parameters such as dynamic strain or vibration which require fast and accurate measurement systems operating in real time. Consequently, advanced distributed fiber-optic sensing schemes for such dynamic measurements have recently attracted significant attention. One possible technique to implement such systems is based on stimulated Brillouin scattering [2]. Although a number of investigations have been made in this area to enhance the speed of the measurement over short distances with high spatial resolution, measurement at long distances is inherently slow [3]. On the other hand, distributed dynamic fiber-optic sensors based on coherent Rayleigh scattering offer interesting alternatives for fast distributed measurements over long distances. Recently, distributed acoustic sensing (DAS), which is the use of an optical fiber to measure vibrations over an extended region, has become a ubiquitous technique [4]. Recent survey indicates that the DAS market has been growing steadily in the past few years and is expected to surpass $\$ 2$ bn by the year 2025 [5]. It has a number of interesting applications including monitoring the safety of assets such as railway infrastructures and oil and gas pipelines.

In this contribution, we provide an overview of some of the recent advances of research in the area of DAS based on phase-sensitive OTDR ( $\phi$-OTDR). The article is organized as follows: Section 2 describes the basic operating principles and physical mechanisms used in $\phi$-OTDR sensing. Section 3 is dedicated to the discussion of some of the recently proposed advanced schemes used to enhance the accuracy of the measurement in $\phi$-OTDR sensors. Then, enhanced configurations which address the critical issue of improving the 
measurement spatial resolution in $\phi$-OTDR are presented in Section 4. This is followed by a discussion of $\phi$-OTDR sensors which extend the speed of measurement beyond the conventional limit set by the round-trip time (RTT) of the sensing fiber in Section 5. Finally, some $\phi$-OTDR configurations to extract additional information for precise quantification of external disturbances, including frequency shift-based measurements and direct detection phase demodulation schemes are discussed in Section 6, and a summary of the review is given in Section 7.

\section{Basic Principle of Distributed Sensing in $\phi$-OTDR}

The most commonly used scheme to implement DAS systems is $\phi$-OTDR, which is based on the observation of coherent Rayleigh backscattering from a sensing fiber in time domain. The operating principle of $\phi$-OTDR can be explained by modeling the sensing fiber as a set of $M$ random scattering elements each with reflectivity $r_{i} \ll 1$, as shown in Figure 1. When light from a coherent pulse reaches each element, it will be backscattered, and since the field contributions from adjacent elements within the spatial length of the pulse have similar phase relations owing to the coherence of the light, the backscattering field from each one will interfere at the receiver. When measured for a duration corresponding to the entire length of the fiber, this backscattering field results in a distributed coherent speckle pattern whose local phase and intensity are sensitive to local disturbances.

The electric field at the receiver $(z=0)$ which is backscattered from the coherent pulse propagating along the fiber is the sum of the contributions of the fields from $M$ random scattering elements within $z \in\left[\left(t v_{\mathrm{g}}-w_{\mathrm{p}}\right) / 2, t v_{\mathrm{g}} / 2\right], v_{\mathrm{g}}$ being the group velocity of light and $w_{\mathrm{p}}$ is the spatial length corresponding to the pulse duration, and is given by $[6,7]$ :

$$
E(t)_{z=0}=E_{0} e^{-2 \alpha \bar{z}} e^{j \omega t} \sum_{i=1}^{M} r_{i} e^{j \phi_{i}}
$$

where $\alpha$ is the loss, $\bar{z}=1 / 2\left[t v_{\mathrm{g}}-w_{\mathrm{p}} / 2\right]$ is the position corresponding to the center of the pulse, $\omega$ is the angular frequency of the incident light, and $\phi_{i}$ is the total phase change of light at the $i$ th scattering element including the phase accumulated by the field in its round-trip travel to scatterer $i$ and back. Note that all length scales shown for position $\mathrm{z}$ are calculated by considering that $t=2 z / v_{\mathrm{g}}$ is the time needed for light to make a round-trip travel over a length of $\mathrm{z}$. This results in the corresponding reverse conversion of $z=1 / 2 t v_{\mathrm{g}}$. When the field in (1) is observed for each location, the change in intensity $\Delta I$ of the photodetected backscattering signal from a static condition with respect to the one corresponding to a perturbation at the $l$ th scattering element can be expressed as follows [8]:

$$
\begin{aligned}
\Delta I & =I_{M}-I_{M_{p}} \\
& =2 \sum_{u=1}^{l-1} \sum_{v=l}^{M} r_{u} r_{v}\left[\cos \left(\phi_{u}-\phi_{v}\right)-\cos \left(\phi_{u}-\phi_{v}-\theta_{p}\right)\right],
\end{aligned}
$$

where $r_{u, v}$ is the reflectivity for positions $\mathrm{u}$ and $\mathrm{v}, \phi_{u, v}$ are the corresponding phases, and $\theta_{p}=0$ for $z<z_{p}$ and $\theta_{p}=\phi_{p}$ for $z \geq z_{p}$, where $z_{p}$ is the perturbation location and $\phi_{p}$ is the phase change due to perturbation. Hence, the local intensity change of the backscattering field is dependent on the phase change at the scattering element which can be induced by any external impact such as vibration or heating which alters either the refractive index or the optical path length at that particular location. A simple distributed measurement over a distance $S$ using this technique is shown in Figure 2(a). Pulses from a coherent source are sent into the sensing fiber through a circulator, which also taps the coherent Rayleigh backscattering signal to a photodiode for subsequent digitization and fast realtime acquisition. Note that this is a simplified schematic as many $\phi$-OTDR configurations include additional components such as modulators, amplifiers, optical filters, and polarization controllers. The maximum repetition rate of the measurement is limited by the round-trip time given by $\mathrm{RTT}=2 S / v_{\mathrm{g}}$, where $v_{\mathrm{g}}=c / n$ is the group velocity of light, $c$ being the speed of light in free space and $n$ the refractive index of the fiber. Note that in many practical scenarios, a number of traces $N_{\text {av }}$ need to be acquired to perform averages and reduce the noise in the raw traces. Together with Nyquist's sampling criterion, the maximum measurable frequency of vibration becomes

$$
f_{\mathrm{vib}}=\frac{1}{2} \times\left(\frac{v_{\mathrm{g}}}{2 S \times N_{\mathrm{av}}}\right)=\frac{1}{4}\left(\frac{v_{\mathrm{g}}}{S \times N_{\mathrm{av}}}\right) .
$$

Hence, this scheme can be used to measure vibrations at high repetition rate and is suitable for distributed dynamic monitoring systems for multiple real-time vibration measurements.

Note that, as will be discussed in Section 4, there are also more recent configurations which sample an external disturbance at faster sampling rates by sending multiple "colored" pulses along the fiber. This can then be used to obtain multiple sampling of each location within a single RTT, thereby surpassing the theoretical limit given in (3) by a factor of the number of frequencies used. In a simple distributed measurement using this technique, a number of raw traces are acquired, and their difference with a reference trace is calculated for the entire length of the fiber. The resulting differential trace set will have nonzero values at points of disturbance while being near zero at locations without disturbance, as shown in Figure 2(b) [9]. The evolution of the change at a disturbed location can then be monitored to extract information such as frequency and amplitude of the external impact. Schemes for the extraction of additional information which cannot be obtained using simple intensity change measurements alone exist, and they enable more precise quantitative measurement of an external impact, as will also be discussed in Section 6. 


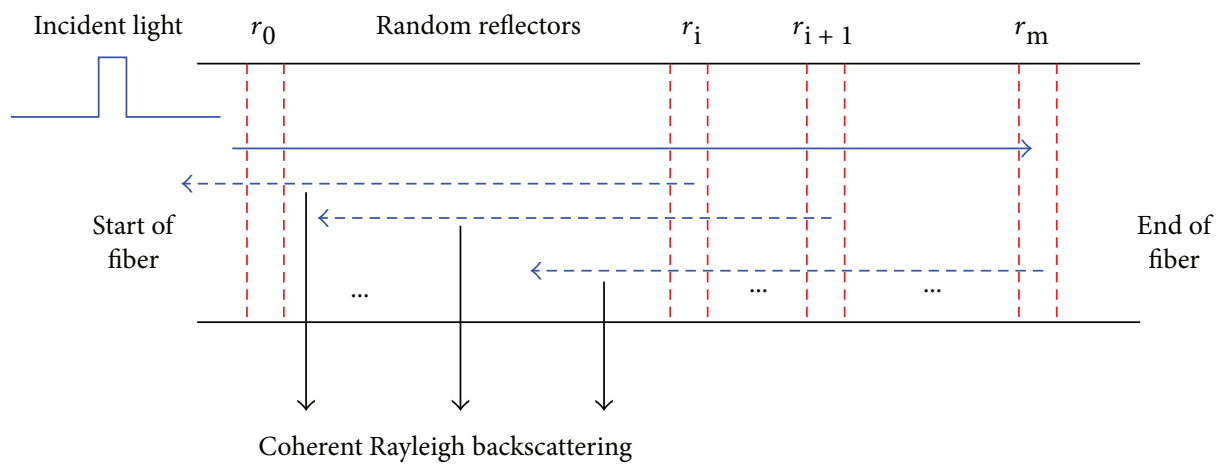

FIGURE 1: Coherent Rayleigh backscattering in a single-mode fiber modeled as a set of randomly distributed scattering elements.

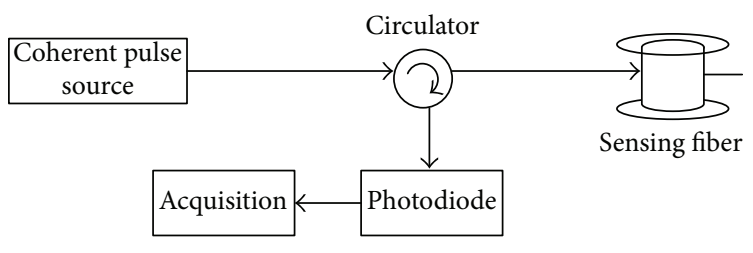

(a)

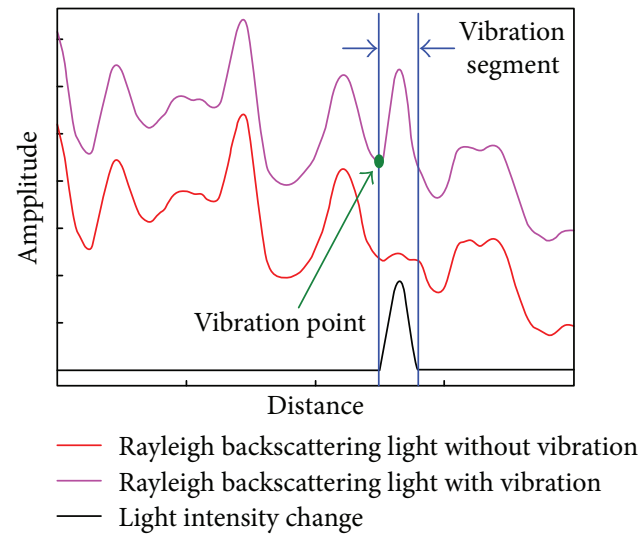

(b)

FIgURE 2: (a) Schematic of a simple $\phi$-OTDR configuration and (b) intensity change in backscattering traces at perturbation location [9].

\section{3. $\phi$-OTDR Sensors for Measuring Change in Backscattering Intensity}

Early implementations of $\phi$-OTDR were focused on measuring the change in intensity and the frequency of the backscattering signal from each location along the sensing fiber to obtain partial information on the disturbance, including its precise location and frequency. The first such implementations involved simple direct detection techniques for measuring intensity changes for intrusion detection systems $[10,11]$. There are also techniques used to enhance the SNR of the backscattered signal in $\phi$-OTDR using moving averaging and moving differential [12]. Some advanced schemes to enhance the SNR of the measurement were proposed and demonstrated employing coherent detection by mixing the backscattering signal with a local oscillator [13]. Since the polarization of the coherent speckles along the sensing fiber cannot be controlled, this scheme necessitates the use of expensive polarization maintaining configurations. Another advanced technique which enhances the SNR of $\phi$-OTDR traces is based on the resolution of the backscattering in wavelet domains [14]. Most of these schemes involve the acquisition of a number of traces to perform accurate measurements and hence can result in a significant reduction of the dynamic performance of the sensor, besides the challenge related to the inevitable accumulation of intermediate data during continuous real-time monitoring.

More recently, proposed methods involve enhancement of the SNR of the backscattered signal, resulting in high visibility by using high extinction ratio (ER) pulses which reduce the coherent noise [7]. In these schemes, the authors analytically show that, for some coherence of the seed laser used to generate the probing pulses, the SNR of the coherent Rayleigh backscattering signals scales with the square root of the extinction ratio of the pulses. This is thanks to the reduction of the coherent noise, which occurs due to the interference of the backscattering signal from the probing pulse with the residual light at the " 0 " level in the same band. Using this method, an experimental demonstration of the measurement of ultrasonic vibrations with frequency up to $39.5 \mathrm{kHz}$ is reported at distance of $1.25 \mathrm{~km}$ with a spatial resolution of $5 \mathrm{~m}$. Other related investigations are focused on the limiting factors involved in $\phi$-OTDR measurement, including that of modulation instability [15], which is introduced due to the use of high-peak-power pulses.

Later studies of techniques to enhance the SNR of the signal include the use of advanced signal processing with optical pulse coding for fast denoising [16]. The method involves sending multiple pulses in the sensing fiber 
instead of a single one and obtaining the single pulse equivalent via a decoding process. This method enables enhanced measurement of the distributed vibration using low-cost DFB lasers having linewidth in the order of few $\mathrm{MHz}$. This is made possible by introducing two coherence conditions which determine the range of the linewidth of the light source used and the bit time, which is the duration between adjacent pulses. These are the intrapulse coherence condition, which requires existence of coherence within the pulse width, and the interpulse incoherence condition, which necessitates that it must be such that there is no coherence between signals in consecutive pulses to avoid interference of the backscattering from adjacent pulses.

The design of the source modulation scheme, which is schematically explained in Figure 3, is done in such a way that both amplified spontaneous (ASE) and coherent noises are reduced and pulses with high ER are generated. Specifically, the CW signal is first used to generate a train of $N$ pulses in the "1" state with bit time of $T_{\text {base }}$, using a Mach-Zhender modulator (MZM). A subsequent acoustooptic modulator (AOM) is then used to apply coding by turning the light on or off with bit duration $T_{\text {bit }}$. Both modulators are used to suppress the "0"-level ASE noise, which is also further reduced in both the " 0 " and " 1 " levels via filtering. Note that the delay $\Delta t$ has been introduced in the modulating RF signals in order to account for the delay introduced by the different locations of the MZM and AOM in the optical setup. Thanks to the high ER of the AOM, the pulses will also introduce reduced coherent noise when used to probe the fiber. The method is used to demonstrate distributed measurement of vibration at a distance of $5 \mathrm{~km}$ with spatial resolution of $5 \mathrm{~m}$. The interesting advantage of this SNR enhancement technique is that no additional optical components are needed, with the only difference from conventional means being the modification of the modulation scheme used to generate the pulses. Note that similar SNR enhancement has been demonstrated in other schemes including standalone BOTDA and Raman distributed temperature sensor (RDTS) and hybrid distributed optical fiber sensor systems in which the fast denoising benefit of optical pulse coding is employed in simultaneously enhancing both temperature and strain measurements [17]. The same method based on pulse coding is also used to demonstrate a cost-effective hybrid distributed acoustic and temperature sensor (DATS) which combines RDTS and $\phi$-OTDR schemes in one by using a shared source, sensing fiber, and intermediate optical components $[18,19]$. The simultaneously obtained temperature resolution using this technique is $0.5^{\circ} \mathrm{C}$ at the same spatial resolution of $5 \mathrm{~m}$.

There has also been investigation of generation of high ER pulses for phase-OTDR using nonlinear Kerr effect [20]. Note that the visibility of OTDR traces for $\phi$-OTDR is dependent on the extinction ratio as has been demonstrated in previous studies [7]. The method in [20] hinges on the observation that a slight variation of the power of a sinusoidally modulated optical signal input to a nonlinear Kerr medium results in a large variation of the sidebands generated by self-phase modulation in the medium.
The authors suggest the use of the schematic shown in Figure 4 for generating the high ER pulses, where a CW signal from NP Photonics FLS-25-3-1550-12 laser is first allowed to pass through an electro-optic modulator (EOM) which generates an optical signal sinusoidally modulated with $8.9 \mathrm{GHz}$ RF signal. Then, a second EOM is used to impose the $50 \mathrm{~ns}$ square pulse on the modulated signal, which is then allowed to pass through a Kerr medium (KM) with $2 \mathrm{~km}$ long dispersion-shifted fiber having a zero-dispersion wavelength of $1552 \mathrm{~nm}$ and undergo self-phase modulation which generates sidebands. When using this source in a $\phi$-OTDR, a couple of filters is employed to suppress the signal carrier and the additional sidebands generated by the EOM [20]. One of the sidebands at the $n$th order is filtered using a narrow optical bandpass filter (BPF). The extinction ratio enhancement in the output optical pulses is higher for higher-order sidebands. The comparison between the backscattering signals obtained from the resulting significantly high ER pulses and those obtained from directly modulated CW light is reported to yield an enhanced SNR while measuring vibration of $1 \mathrm{kHz}$ at $500 \mathrm{~m}$. However, no quantitative evaluation of this enhancement is provided except the qualitative comparison of the raw traces for different lengths of the sensing fiber.

Real-time measurement of vibrations has also been done using hardware implementations at the receiver. For instance, a distributed fiber-optic microphone system is implemented using a $\phi$-OTDR scheme with a sample and hold circuit, as shown in Figure 5 [21]. The circuit can be used to tune the sensing location so as to "listen" to a particular point in the fiber and recover the sound in real time. Even though the method cannot be deemed fully distributed as it enables a single real-time measurement at a time, the technique is used to detect and reconstruct generic vibration signals including human voice, with a spatial resolution down to $60 \mathrm{~cm}$ over a distance of $300 \mathrm{~m}$. In the same study, the use of high numerical aperture fibers is also verified to increase the intensity of the detected coherent Rayleigh scattering at the receiver and enhance the SNR of the measurement. Comparison of real and detected human voices resulted in some discrepancy as the scheme, which uses simple direct detection with no phase demodulation, cannot be used to determine the phase change of the optical signal due to the disturbance.

Loranger et al. reported a method to enhance the Rayleigh backscattering signal for improved SNR measurement in $\phi$-OTDR systems [22]. The system is based on a costeffective method with reduced fabrication complexity based on exposing a single-mode fiber to UV light without requiring critical alignment compare to the writing of multiple FBGs. The method yields enhanced SNR due to an order of magnitude higher increase in the detected backscattering amplitude.

One of the issues concerning $\phi$-OTDR sensors is modulation instability. Some techniques have been proposed to address this issue including making the shape of the probing pulse the main focus of the design [23]. According to the investigation in this work, from among four different types of pulse shapes for the same total pulse energy including 


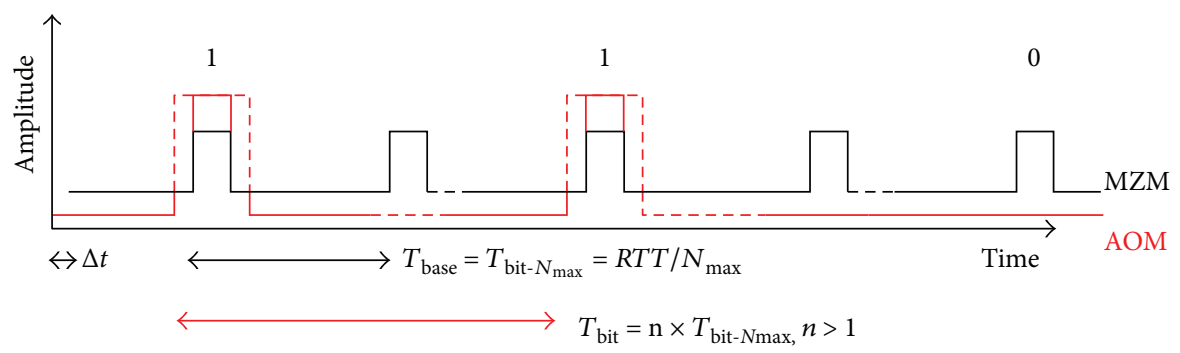

FIGURE 3: Design of the modulation scheme for a high-performance $\phi$-OTDR sensor.

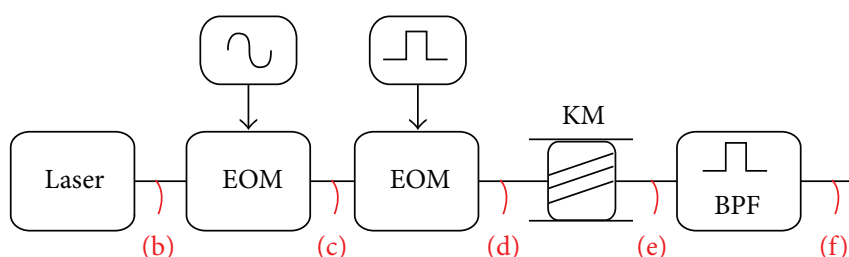

(a)
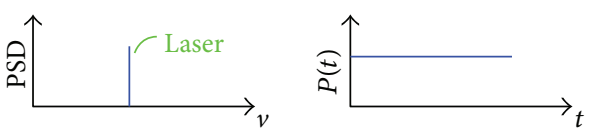

(b)

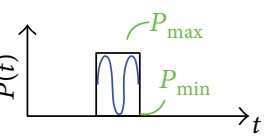

(d)

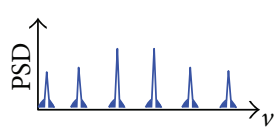

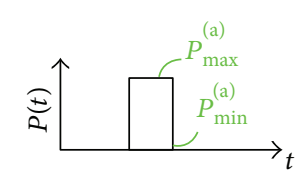

(e)

(f)

FIGURE 4: Generation of high ER pulses for a high SNR $\phi$-OTDR using the nonlinear Kerr effect [20]: (a) schematic of the generation, (b) CW light from laser, (c) sinusoidally modulated signal, (d) signal after pulse modulation, (e) signal after nonlinear Kerr medium, and (f) after filtering the nth order sideband $(P(t)$ in all cases stands for the power of the signal).

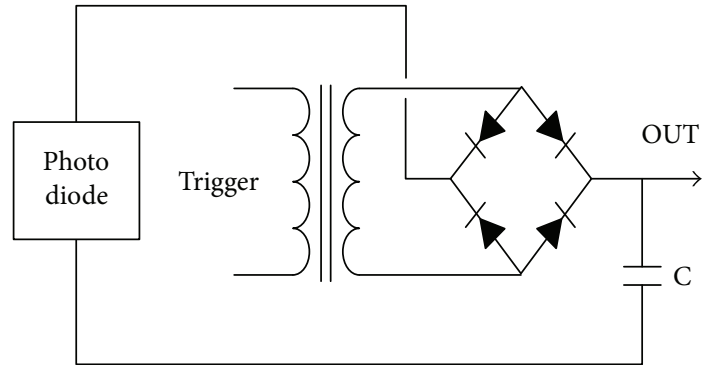

Figure 5: Sample and hold circuit used to retrieve vibration at a particular point in the sensing fiber [21].

triangular, rectangular, Gaussian, and super Gaussian, using triangular or Gaussian-like pulses reduces visibility fading in received $\phi$-OTDR traces and results in longer sensing range or higher sensitivity. Rectangular pulses are shown to exhibit more significant degradation in visibility, especially at long sensing distances. An SNR enhancement method for a $\phi$-OTDR sensor based on empirical mode decompression (EMD) method has also been proposed and experimentally demonstrated [24]. It is based on the decomposition of a noisy signal into the sum of a number of intrinsic mode functions (IMF) and a residual component. The calculation of the IMF functions is performed using an algorithm called sifting, and they are shown to exhibit unique characteristics such that the frequency gradually decreases from the first to the last IMF. The high frequency noise will be concentrated on the first few IMFs, and this feature enables noise reduction via either filtering or thresholding. In the proposed scheme, the correlation between each IMF and the raw signal is determined using the Pearson correlation coefficient (PCC). Using the method together with EMD in a scheme called PCCEMD, for a sensing distance of $2 \mathrm{~km}$ and spatial resolution of $5 \mathrm{~m}$, the reported improvement on the SNR during measurement of a vibration of $1.2 \mathrm{kHz}$ is a significant amount of $\sim 40 \mathrm{~dB}$. The combined EMD-PCC method is reported to yield noise reduction significantly higher than both the one without use of PCC and other methods such as wavelet denoising [12]. Note that the sifting algorithm used in the denoising, which is performed offline in the digitally acquired signal, can incur significant processing overhead which may limit the dynamic performance of the technique.

In [25], the use of distributed fiber-optic sensors for future audio and acoustic applications is suggested, and a 
method to detect acoustic signals in air, similar to the one with a microphone, is proposed. In particular, attaching a bare sensing fiber in a $\phi$-OTDR sensor to a polysterene sheet is shown to improve the sensitivity of the fiber to acoustic signals applied through air. Experimental results show the detection of low intensity acoustic vibrations applied using a speaker placed at a distance of $30 \mathrm{~cm}$ away from the fiber. The sound source and sensing fiber were placed in a reverberation chamber which generates a large sound pressure level (SPL), and measurements of acoustic signals from 0.5$1.75 \mathrm{kHz}$ are demonstrated with detection of sound signals at naturally occurring ranges of $108-115 \mathrm{~dB}$ SPL, with a spatial resolution of $1 \mathrm{~m}$. Note that the unit $\mathrm{dB} S \mathrm{SP}=20 \mathrm{log}$ $\left(p / p_{0}\right)$, where the measured sound pressure is $p$ in Pascal $(\mathrm{Pa})$ and the standard reference pressure $p_{0}=10^{-5} \mathrm{~Pa}$, is employed in quantifying and comparing the pressure due to different acoustic signals $[26,27]$.

\section{DAS Sensitivity and Configurations for High-Speed Vibration Measurements beyond Conventional Limits Set by the Sensing Distance}

In DAS systems, the measurement of sensitivity is key to the comparison of the performances of individual systems. To this end, some investigations have been made including [28], where the authors propose the definition of DAS sensitivity as the minimum input signal which generates an $S N R \geq 1$, in which the induced dynamic phase change is measured with a specific fraction $\rho$ of the virtual microphone. The sensitivity is defined using the following relationship [28]:

$$
\left.\sigma\right|_{\min }=\sqrt{\frac{z_{\rho}}{2 \mathrm{SNR}_{\mathrm{s}}}},
$$

where $\left.\sigma\right|_{\min }$ is the standard deviation of the dynamic phase signal which also determines the minimum measurable amplitude, $z_{\rho}$ is a dimensionless parameter obtained from the Rayleigh distribution, and $\mathrm{SNR}_{\mathrm{s}}$ is the static signal-tonoise ratio obtained from a single measurement of backscattering signal at the receiver. In other words, the sensitivity is the minimum input signal for which the dynamic signal-tonoise ratio $\mathrm{SNR}_{\mathrm{d}}$ is such that $\operatorname{Prob}\left\{\mathrm{SNR}_{\mathrm{d}} \geq 1\right\}=\rho$. Experimental evaluations of this specification are made on a phase measurement system employing a $90^{\circ}$ hybrid and coherent detection involving $1 \mathrm{~km}$ single-mode fiber and three probing pulses with durations 25, 50, and $100 \mathrm{~ns}$. Accordingly, for increasing pulse width, the $\mathrm{SNR}_{\mathrm{s}}$ increases while, since $\rho$ increases, a higher fraction of the virtual microphones is required for a better performance, which in turn degrades the sensitivity.

Another venue in which investigation has been done is in extending the measurable vibration frequency in a $\phi$-OTDR beyond the conventional limit set by the round-trip time (RTT) given by (3). To this end, a system which uses multiple pulses having different frequencies within a single RTT has been demonstrated [29]. The principle of operation is illustrated in Figure 6, where in the left side acquisitions of backscattering traces from pulses having multiple "colored" frequency components are used to probe the fiber, with the element $A_{i j}$ representing the amplitude of the $i$ th pulse at the $j$ th acquisition. The right side of Figure 6 shows the sampling for a particular position along the fiber, which is equivalent to taking multiple samples corresponding to each colored pulse at every spatial point within one RTT.

If the number of multiple-frequency pulses is $n$ within a round-trip time of RTT, the sampling period which in conventional sensors has a minimum value of RTT, becomes R $\mathrm{TT} / n$ in this case, enabling a proportional increase in measurement speed. At the receiver, short-time Fourier transform (STFT) with a duration corresponding to the spatial resolution is applied to the backscattering signal from pulses of different frequencies.

This scheme is demonstrated with 16 frequency-encoded pulses and coherent detection using a balanced photodiode with the dummy-frequency component used between each pulse being outside the detection bandwidth of the photodiode. Along a sensing distance of $5 \mathrm{~km}$ and with a spatial resolution of $20 \mathrm{~m}$, the reported vibration frequency measured using this technique is $80 \mathrm{kHz}$. This is beyond the theoretical limit of the measurable vibration frequency in conventional schemes using only a single frequency, which would be, considering a maximum repetition rate of $20 \mathrm{kHz}$ and the Nyquist criterion, $10 \mathrm{kHz}$ for a sensing distance of $5 \mathrm{~km}$.

Here, it is worth making a comparison of the above method with a recently proposed technique which improves the speed of the measurement using a novel configuration based on frequency division multiplexing of pulses sent to the sensing fiber within one RTT [30]. The schematic is shown in Figure 7, which is also an enhancement of earlier methods proposed in $[31,32]$. The rate of measurement compared to conventional single-frequency $\phi$-OTDR is increased by the number of linearly frequency modulated (LFM) probe pulses generated using an arbitrary waveform generator (AWG) driving an AOM. The backscattering from different frequency ranges is separately demodulated by a matched filter algorithm at the receiver. The method, which also uses a coherent detection at the receiver to determine the phase change, is used to demonstrate the measurement of vibrations of up to $9 \mathrm{kHz}$ at a spatial resolution of $10 \mathrm{~m}$ over a sensing distance of $24.7 \mathrm{~km}$. The enhanced method proposed here also addresses the issue of necessity to use high bandwidth receiver to detect the backscattering due to the chirped pulses in the AOM in $[31,32]$ by using undersampling. Note that this is a new configuration which has the attributes of both conventional frequency and time domain configurations due to the use of frequency-modulated probe pulses.

\section{5. $\phi$-OTDR Configurations for Distributed Dynamic Measurements with High Spatial Resolution}

Typically, the spatial resolution of $\phi$-OTDR sensors has been in the order of a few meters, with most demonstrations thus far being 5, 10, and even $20 \mathrm{~m}$. Recently, dynamic distributed 

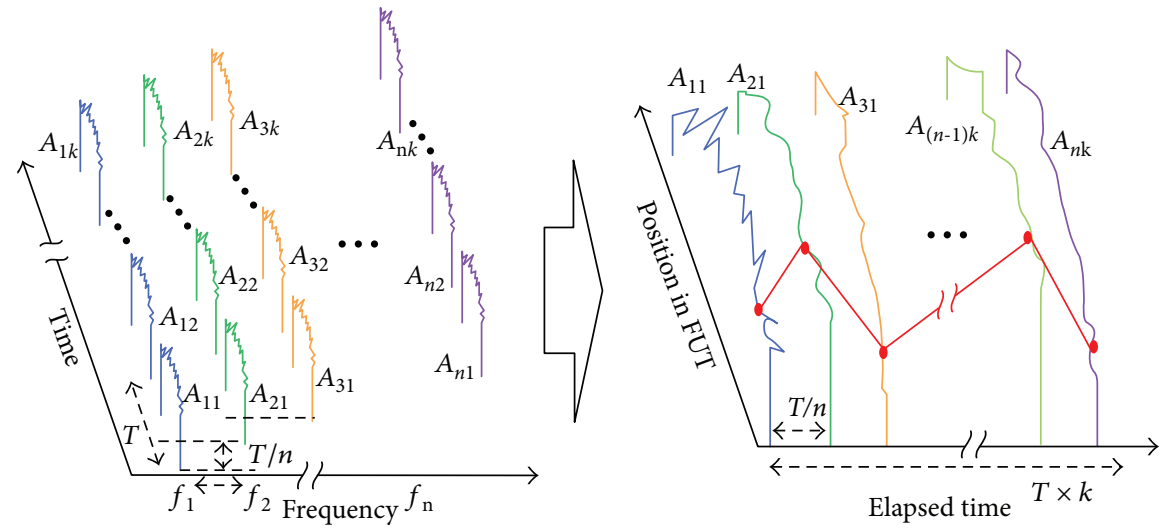

FIGURE 6: Schematic of arrangement of traces in a multiple-frequency $\phi$-OTDR for measurement of vibrations below a sampling time of RTT [31].

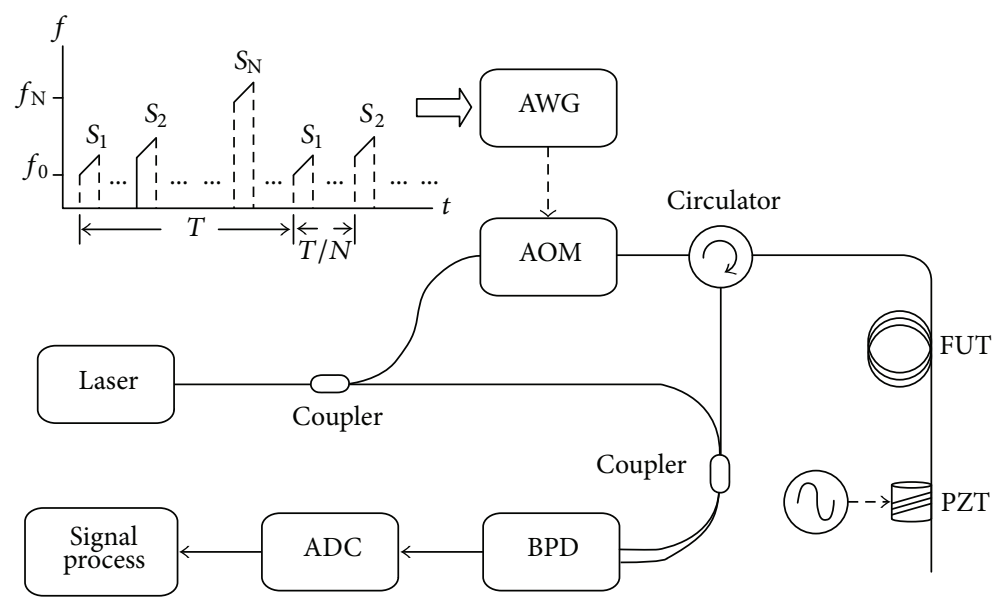

FIGURE 7: Experimental setup of $N$ LFM pulses $S_{\mathrm{i}}$ within a time $T$ slightly longer than RTT for distributed vibration measurement of vibration frequencies with sampling times of fractions of RTT of the fiber [30]. AWG: arbitrary waveform generator; AOM: acousto-optic modulator, BPD: balanced photodiode.

sensors based on $\phi$-OTDR for the measurement of strain at high spatial resolution have been under investigation. A configuration based on the concept of chirped pulse amplification is used for SNR enhancement in a $\phi$-OTDR sensor as reported in [33]. This scheme enables measurements at small pulse width, resulting in SNR enhancement at spatial resolution values down to $1.8 \mathrm{~cm}$. As shown in the experimental setup used to demonstrate this technique shown in Figure 8 , an initial dispersive medium based on a linearly chirped fiber Bragg grating (LC-FBG) with a given dispersion coefficient is used to stretch the pulse.

At the receiver, the backscattering signal is allowed to pass through another LC-FBG, with the opposite dispersion coefficient. The net effect of this procedure is that, while the SNR will be enhanced, the system will retain the spatial resolution corresponding to the initial input pulse before it is stretched due to the opposite dispersive operation which takes place in the optical domain. The experimentally reported sensing distance in this contribution is only $8 \mathrm{~m}$ owing to the pulse repetition rate of the mode-locked laser used to generate the narrow coherent pulses. The technique requires a high bandwidth at the receiver, with components used in the demodulation being a $35 \mathrm{GHz}$ photodiode and a digitizer with a sampling rate of $80 \mathrm{GS} / \mathrm{s}$.

Most notably, an interesting technique to implement $\phi$-OTDR with enhanced spatial resolution which is decoupled from the probing pulse width has also been proposed and demonstrated, using swept-frequency pulse [34]. The technique is based on the principle used in optical pulse compression reflectometry (OPCR) which uses frequencymodulated pulse compression. LFM optical pulses are used with temporal width large enough to obtain higher SNR in the backscattering signal, and a matched filter is used at the receiver to obtain the desired low spatial resolution.

The method implemented for a $\phi$-OTDR system is shown in Figure 9, where the signal from a narrow linewidth RIO laser is first modulated using the EOM driven by a voltage-controlled oscillator (VCO) to sweep the frequency. The LFM optical signal is then injection locked with a distributed feedback laser diode (DFB-LD) in order to enhance the sideband suppression ratio and the output power. The signal is then modulated using an AOM, and the resulting frequency modulated pulses are amplified and sent into the FUT. 


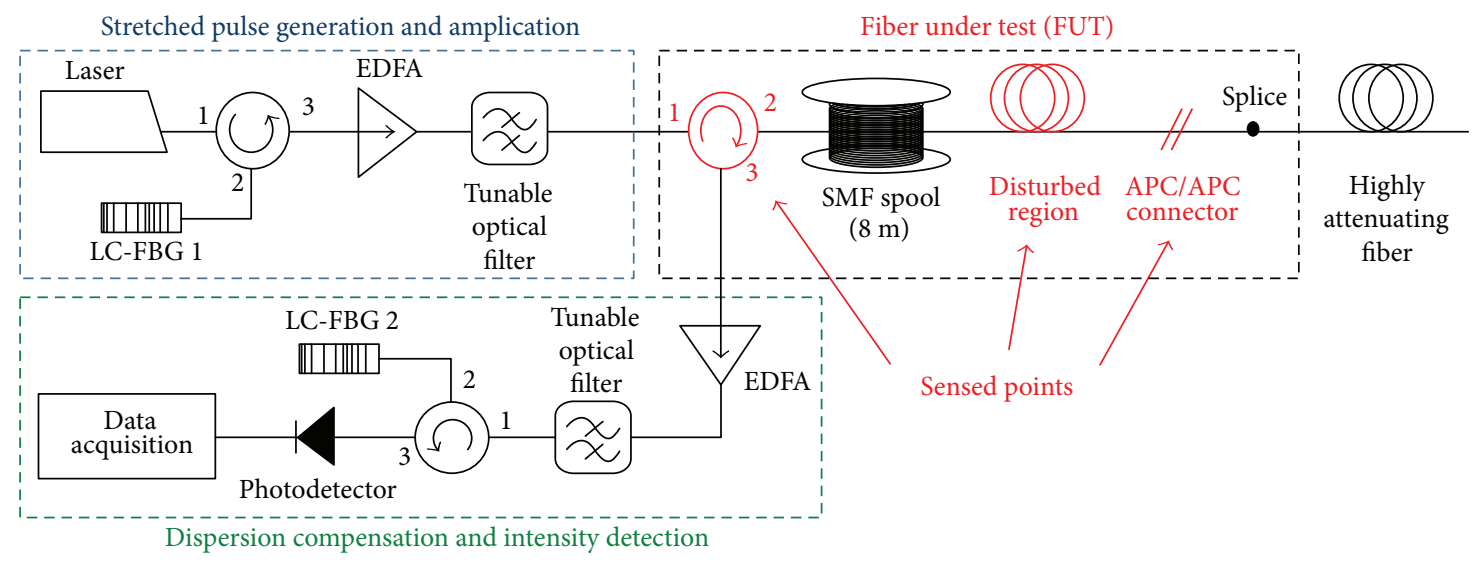

FIGURE 8: Experimental setup for demonstration of SNR enhancement via chirped pulse amplification [33].

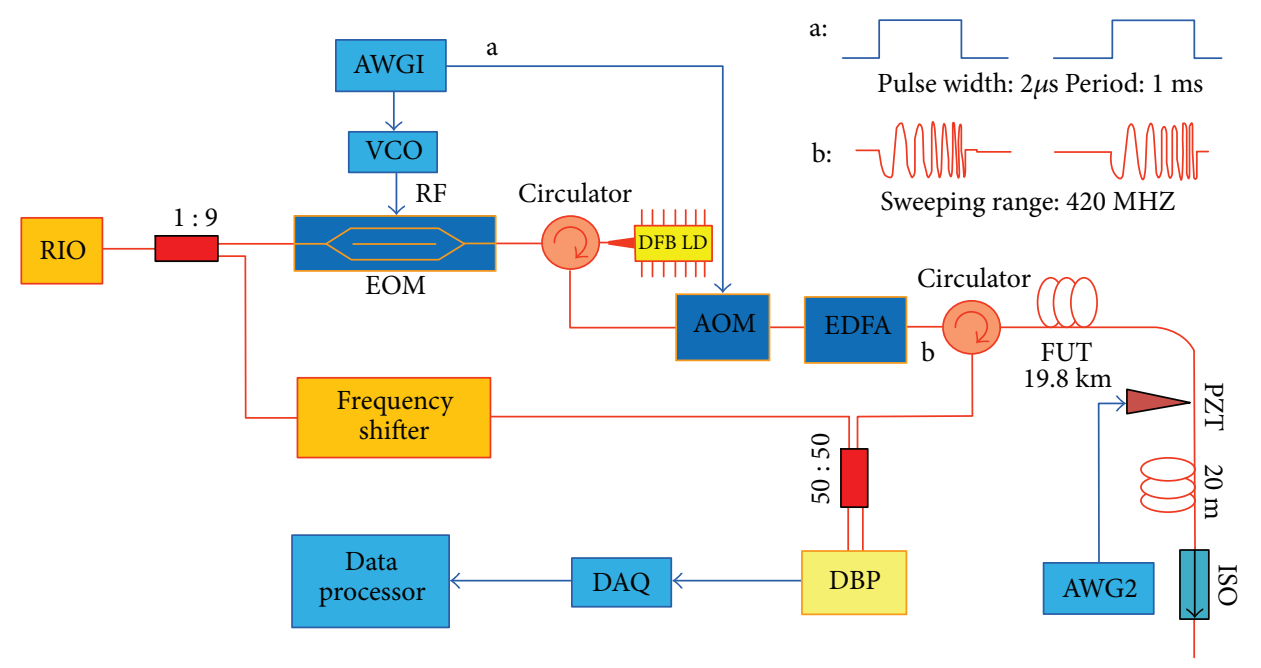

FIGURE 9: Schematic of a high spatial resolution $\phi$-OTDR using a frequency-swept pulse [34].

At the receiver, a double-balanced photodiode (DBP) is then used to detect the coherent mixing of the backscattering with the local oscillator which is split from the seed CW laser. The signal is digitized and acquired using the data acquisition (DAQ) system before it is allowed to pass through a matched filter. The difference between two adjacent locations is used to retrieve the perturbation signal in this scheme. It is worth mentioning that the authors use only the moving differential technique to determine the phase change. However, this can only be used to determine the change in amplitude of the backscattering due to a disturbance, unless a technique similar to the one given in [35] is used. Nonetheless, theoretical analysis shows that measurement using a matched filter is as though the original LFM pulse is compressed as a narrow sinc-like pulse. In this technique, the spatial resolution $R$ (defined as FWHM of the processed signal in this particular demonstration) is given as follows [34]:

$$
R=\frac{c}{2 n B}
$$

where $c$ is the speed of light in free space, $n$ is the refractive index, and $B=K T$, where $K$ is the slope of the LFM and $T$ is the duration of the frequency sweeping range, effectively decoupling the spatial resolution of the measurement from the pulse width. An experimental demonstration with a measurement of a $200 \mathrm{~Hz}$ disturbance at $19.8 \mathrm{~km}$ single-mode fiber is reported with a high spatial resolution of $30 \mathrm{~cm}$ and an SNR of $10 \mathrm{~dB}$, while a large pulse width of $2 \mu \mathrm{s}$ is used to probe the fiber.

Another interesting feature of this method is that it clearly addresses the issues of dynamic range when using narrow pulses, and more importantly, it tackles issues arising from nonlinearity during measurements at long distances when higher peak power levels are used to increase the energy of narrow pulses. The required bandwidth for this particular demonstration is $800 \mathrm{MHz}$ for the photodiode and $2 \mathrm{GS} / \mathrm{s}$ for the DAQ. The spatial resolution performance limit of the proposed sensor is coupled with the frequency sweeping range which also determines receiver bandwidth where as expected, more spatially resolved measurements require higher receiver bandwidth. 


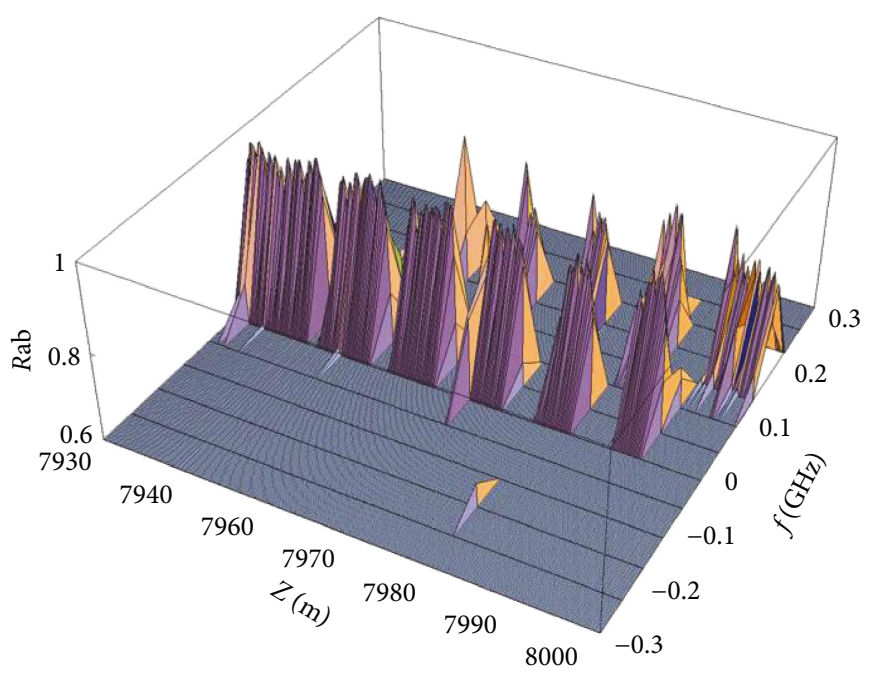

(a)

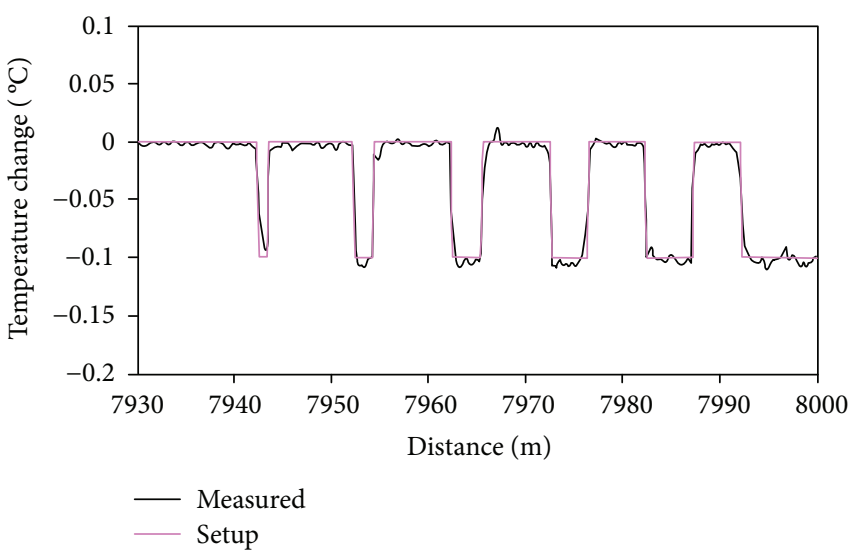

(b)

FIgure 10: (a) Cross-correlation of backscattering intensity traces at different spatial points in scanned pulse $\phi$-OTDR showing peaks corresponding to compensating frequency shifts and (b) measurement of distributed temperature change [36].

\section{6. $\phi$-OTDR Sensors for Precise Impact Extraction Using Frequency Shift and Phase Demodulation Techniques and Addressing Polarization Fading}

$\phi$-OTDR sensors involving measurement of intensity changes offer information on the location, frequency, and amplitude of the backscattering signal. However, a more precise quantitative measurement of an external disturbance requires additional information on the change in signal behavior induced by strain or temperature changes. To this end, techniques which are based on frequency shift measurements [36-39] and others using direct demodulation of the phase change [35, 40-44] have been proposed.

The frequency shift method for external impact extraction using $\phi$-OTDR is based on the observation that the frequency of the probing pulses can be matched with a corresponding change in temperature or strain with some proportionality constant. For a source frequency shift of $\Delta v$, a strain change of $\Delta \varepsilon$ and a temperature change of $\Delta T$ are calculated as follows [36]:

$$
\begin{aligned}
& \frac{\Delta v}{v_{o}}=-6.92 \times 10^{-6} \Delta T, \\
& \frac{\Delta v}{v_{o}}=-0.78 \Delta \varepsilon,
\end{aligned}
$$

where $v_{o}$ is the nominal frequency of the probing light. Measurement of external impact using this method involves an initial acquisition of multiple sets of traces (including averaging) while scanning the frequency of the probing pulses. This is followed by performing cross correlation $R_{\mathrm{ab}}$ of the traces acquired at multiple-intensity traces $X_{\mathrm{b}}$ with a reference trace $X_{\mathrm{a}}$ as shown in Figure 10(a). For points in which there are no disturbances, the calculated correlation has a peak only at the reference frequency while heating/cooling the fiber results in the peak occurring at another frequency. The offset of the frequency of the peak with that of the reference trace is used in (4) to calculate the temperature or strain, as shown in Figure 10(b) for temperature change measurement. This method has been used to demonstrate temperature sensing with a resolution down to $0.01 \mathrm{~K}$. However, the method, which uses slow frequency scanning, is quasi-static and cannot be used for measurement of dynamic perturbations.

To address this issue, another configuration which employs the same principle has been proposed, this time based on sending the equivalent of multiple-frequency pulses on a single shot through chirping $[37,38]$. In this method, the duality of the temperature and strain changes with respect to the source frequency change is utilized to measure both dynamic strain and temperature. As shown in the experimental setup depicted in Figure 11, the chirp in the singleshot $\phi$-OTDR is generated using a laser diode (LD) whose frequency is varied by applying ramp control input voltage from the signal generator in synchronism with the pulses which drive the semiconductor optical amplifier (SOA) as a modulator.

A distributed measurement using this technique involves calculation of the cross-correlation of traces to determine their longitudinal shift. The measured shift is directly proportional to the amount of temperature or strain, and the slope of the linearity is inversely proportional to the chirp slope, which is a feature enabling the tuning of the sensitivity of the sensor by merely acting on the chirp slope. The traces at the perturbed position are shifted longitudinally with respect to the static condition as shown in Figure 12.

The method has also been used to measure dynamic strain using the relation for strain change given in (4). Using this technique, measurement of temperature with resolution in the order of $\mathrm{mK}$ and that of strain with $4 n \varepsilon$ resolution are reported at a spatial resolution of $10 \mathrm{~m}$ [38]. It is worth noting that, in this scheme, the dynamic range of the measurement which is determined by the total frequency change, 


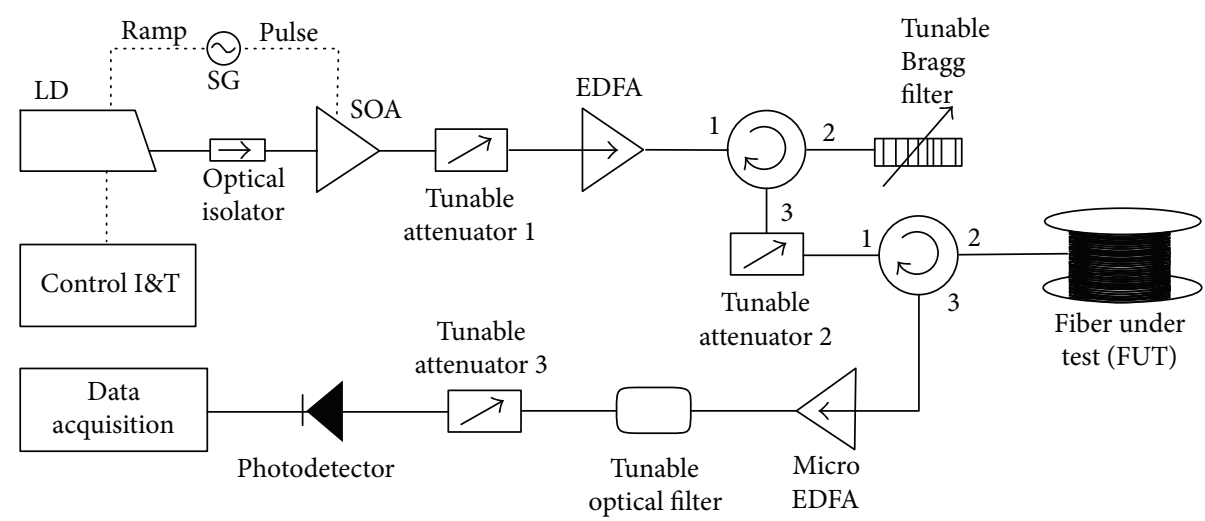

FIGURE 11: Experimental setup of a chirped pulse $\phi$-OTDR sensor [38].

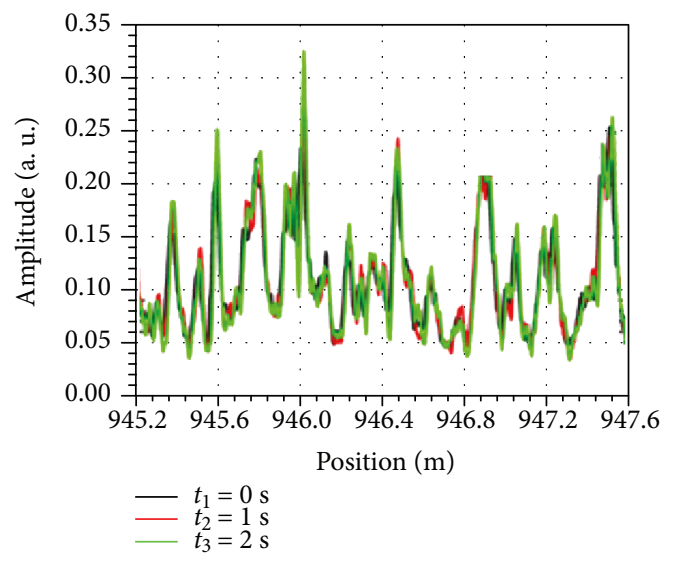

(a)

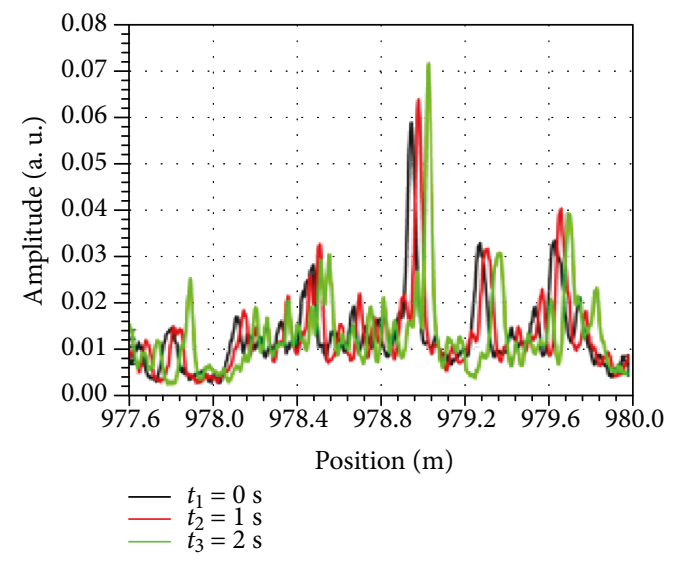

(b)

FIGURE 12: Traces at a segment of the sensing fiber showing: (a) static conditions and (b) longitudinal shift with temperature change at different instances due to heating [38].

is linked to the pulse width and hence to the spatial resolution. In addition, the receiver uses a photodiode with a high bandwidth of $13 \mathrm{GHz}$ and an $\mathrm{ADC}$ with a sampling rate of $40 \mathrm{GS} / \mathrm{s}$ for the reported spatial resolution of $10 \mathrm{~m}$. Note that, owing to its fast measurement capability, the singleshot method has also been used to demonstrate the measurement of high frequency vibrations close to limits set by the sensing distance.

More recently, a dual-frequency $\phi$-OTDR system for measurement of the relative change of physical quantities has been proposed and experimentally demonstrated [39]. In this scheme, a narrowband DFB laser is used as a seed laser to generate consecutive pulses with known frequency shift for each individual measurement. The principle is based on the correlation of the backscattering signals from the two sets of traces for any spatial location. The relative time shift of the backscattering signals, which is obtained by first calculating the peaks of the correlation of the backscattered trace with its shifted version, is then used to determine the local disturbance. In the particular experiment, the change in temperature due to heating of a short segment of fiber at the end of $1 \mathrm{~km}$ has been measured at a spatial resolution of $1 \mathrm{~m}$. Even though dynamic strain measurements are not reported in this particular contribution, monotonic and sign-changing gradient of temperature changes are demonstrated to yield results which are consistent with a commercial temperature measurement device. The proposed system also avoids the coupling of the spatial resolution and dynamic range of the measurement. Besides, shifting of the frequency does not require additional optical components as a simple modulation of the frequency of the DFB laser suffices, and the receiver is based on a simple pin photodiode with $125 \mathrm{MHz}$ bandwidth.

One of the first schemes for direct demodulation of distributed phase change involves digital coherent detection to extract the phase of the coherent Rayleigh traces in the digital domain [45]. Another notable technique for phase demodulation for dynamic strain measurement in a $\phi$-OTDR is based on a $3 \times 3$ coupler demodulation scheme [40]. There is a delayed interferometric system which is kept in thermal isolation at the input of the $3 \times 3$ coupler and three photodiodes in synchronous operation at the three outputs of the coupler. The introduction of a perturbation at any point on the fiber introduces a phase change which will be observed at the input of the interferometer at a resolution determined by the relative delay between the two arms and 


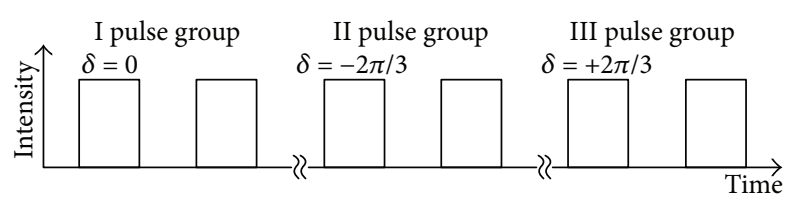

(a)

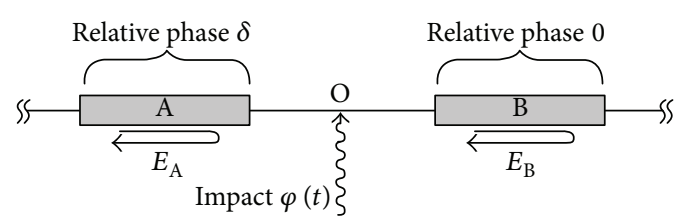

(b)

FIGURE 13: Operating principle of a dual-pulse $\phi$-OTDR scheme: (a) phase modulation in the probing pulses and (b) phase change at a location with external impact traversed by a pulse pair [42].

detected after manipulation of the three signals at the output after photodetection. The technique is used to demonstrate dynamic measurement of strain varying at $5 \mathrm{kHz}$ over a $1 \mathrm{~km}$ distance with a minimum detectable strain range of $80 n \varepsilon$ [40].

This scheme has recently been improved in terms of the performance in both the spatial and frequency resolutions [41]. The later configuration involves the use of a single FBG to filter the ASE noise both in the propagating pulses and the backscattering signal. This scheme is shown to be more efficient than the use of two separate FBGs, as the single FBG can be made to be narrow without facing issues of matching the central wavelength. The method is used to make measurements of dynamic perturbations of up to $3 \mathrm{kHz}$ at a distance of $5 \mathrm{~km}$ with a frequency resolution which can be reduced to $5 \mathrm{~Hz}$. Note that a fringe counting algorithm is used in this scheme for phase unwrapping which inevitably incurs additional postprocessing overhead, which will become multidimensional in a distributed scenario involving a number of points. In addition, the need for thermal isolation of the interferometer and use of three independent photodiodes which must be operated in synchronism render the receiver of this scheme relatively more complex than other alternatives.

A $\phi$-OTDR sensor, which emulates the $3 \times 3$ coupler used in $[40,41]$ and resolves the receiver multiplicity and synchronization issues by making alternate use of a single photodiode and addressing drawbacks of interferometric stability with the use of a double-pulse scheme, has also been proposed [42]. The modulation of the pulses and the operating principle of the configuration is depicted in Figure 13. Each of the three pulse pairs is such that the relative phase-shift $\delta$ is $0,2 \pi / 3$ or $-2 \pi / 3$ as shown in Figure 13(a). Any disturbance which happens at a particular location of the fiber introduces a phase change in the backscattering field $E_{B}$ coming from leading pulse from the pair and not in $E_{A}$ coming from the trailing one as shown in Figure 13(b). At the receiver, the fields from each pulse in one pair will interfere with each other, and the resulting fields from the three interferences will be used to extract the phase change, the same way as in the passive $3 \times 3$ coupler.

Note that, due to the required waiting time for three pulses for a single measurement, the measurable frequency of vibration is reduced by a third. Another method which is not limited by this reduction in measurement bandwidth has been implemented using only a single dual-pulse pair in which a relative frequency shift is introduced between the pulses. An electrical I/Q mixer with a local oscillator with frequency corresponding to the offset is then used to extract the two orthogonal components of the phase, together with unwrapping for phase values outside the range of the arctan function [43]. Both techniques are shown to accurately demodulate the phase change induced by a dynamic vibration with modulated amplitude.

Another more recent phase demodulation technique which does not require coherent detection has also been proposed utilizing the sum and difference of the intensity of backscattering signals from two adjacent points [35]. This technique has been demonstrated both for the case of singleand double-pulse probes. When a certain disturbance occurs at a particular location in the fiber, two backscattering intensity signals are taken and used to retrieve the phase. The authors show that the envelope fitting of the sum, $\operatorname{Sum}^{\prime}(t)$ and that of the difference, Diff $f^{\prime}(t)$ of the two signals for both single- and double-pulse cases are proportional, respectively, to the cosine and sine of the phase, such that

$$
\begin{aligned}
& \operatorname{Sum}^{\prime}(t)=\cos \left(\theta(t)+\frac{\psi_{1}+\psi_{2}}{2}\right), \\
& \operatorname{Diff}^{\prime}(t)=\sin \left(\theta(t)+\frac{\psi_{1}+\psi_{2}}{2}\right),
\end{aligned}
$$

where $\theta(t)$ is the time changing phase and $\psi_{i}(t), i=1,2$ is the relative phase drift at the two adjacent positions 1 and 2. The $\theta(t)$ due to a particular perturbation is obtained merely as the arctan of the ratio of the sum and difference signals. In this particular demonstration, the technique is used to measure dynamic phase changes with speeds of up to $200 \mathrm{~Hz}$ at distance of $182 \mathrm{~m}$ with a spatial resolution of $20 \mathrm{~m}$.

Another phase extraction method employs coherent homodyne detection using a $90^{\circ}$ hybrid and a local oscillator to extract the phase [44]. The basic schematic is shown in Figures 14(a) and 14(b), where the backscattering signal is fed to the input of the $90^{\circ}$ hybrid together with the local oscillator to obtain the two orthogonal components. The reported demonstration using this method yields the demodulation of up to $600 \mathrm{~Hz}$ signal at a distance of $12.56 \mathrm{~km}$ with resolution of $10 \mathrm{~m}$.

It is worth noting that, as has been mentioned above, most conventional techniques for phase demodulation including the use of $90^{\circ}$ hybrid involve phase unwrapping for values of the phase beyond the range of the arctan function. Phase unwrapping, especially in the presence of fast and abrupt changes of phase, is a computationally costly process, and in the case of distributed sensing, it involves multidimensional computations the same as the ones necessary in image processing [46-48]. Note also that the dynamic range 


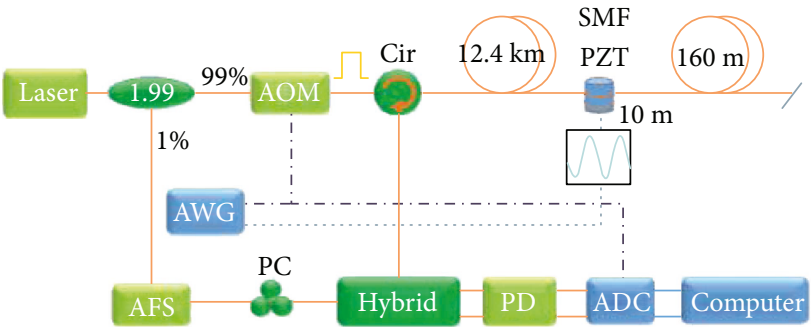

(a)

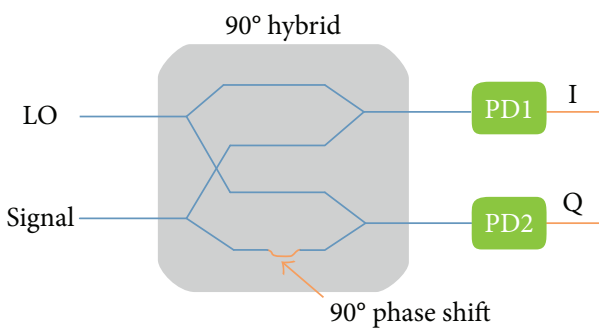

(b)

FIGURE 14: Homodyne demodulation using an optical hybrid [44]: (a) experimental setup using coherent detection and (b) mixing with a local oscillator to resolve I and Q components using an optical hybrid (AFS: acoustic frequency shifter).

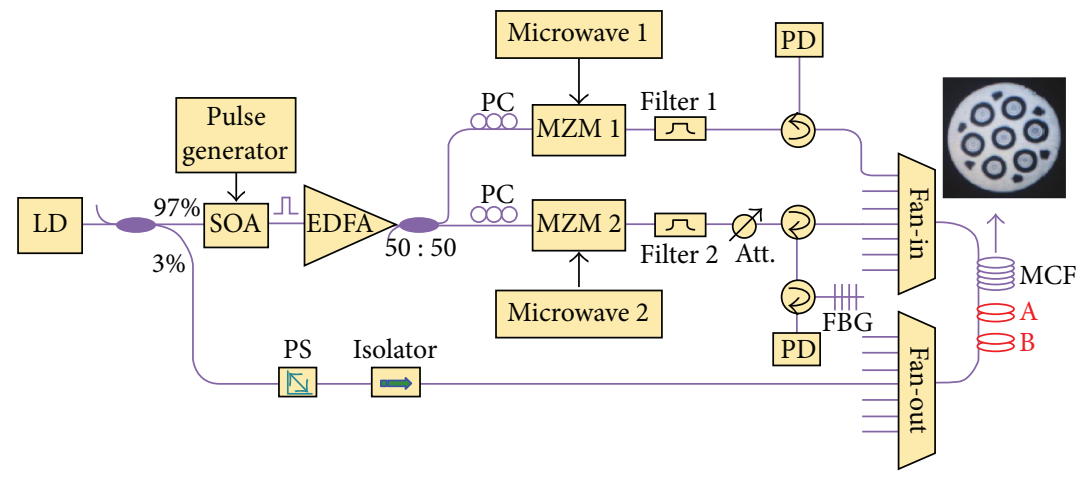

FIGURE 15: Experimental setup of a high dynamic range distributed fiber-optic sensor for static stain/temperature sensing [49].

of temperature measurement using the $\phi$-OTDR configuration for precise impact extraction using frequency shift method demonstrated in [36] is limited. This is due to the fact that the temperature dependence of the property of Rayleigh scattering traces, while allowing for a high temperature resolution down to $\mathrm{mK}$ range, is valid only for small temperature ranges, as the refractive index is also affected at larger ones.

On the other hand, distributed temperature sensing using the BOTDA scheme allows measurement over a large temperature range while temperature resolution is compromised, typically being limited to orders of a few Kelvin at long distances. There is a recently proposed configuration which addresses this issue by using a multicore fiber (MCF) as the transducer in a hybrid configuration combining $\phi$-OTDR and BOTDA [49], which is shown in Figure 15. The two schemes are implemented in two (central and outer) cores of the MCF with a shared pump for both the Rayleigh and Brillouin sensors and fan-out systems to couple the signal to and from the single-core fibers in the components and the MCF transducer. The frequency scanning of the BOTDA is done in the probe while that of the $\phi$-OTDR is performed in the pump, as shown in the experimental setup.

This configuration harnesses the important features of the two sensing systems in a single one as shown in the experimental results depicted in Figure 16. The two sections $\mathrm{A}$ and $\mathrm{B}$ of the sensing fiber are heated, respectively, with small and large temperature changes. The temperature profile using BOTDA given in Figure 16(a) cannot resolve the small changes in section A while the large ones in section
$\mathrm{B}$ are clearly visible. In contrast, the profile made using the $\phi$-OTDR measurement shown in Figure 16(b) is able to resolve the small changes in section $\mathrm{A}$, while for section $\mathrm{B}$ the different measurements are indistinguishable from each other thanks to reduced resolution due to the effect of large temperature changes on the refractive index. Note that this configuration can potentially be used to implement a DATS system for simultaneous, cospatial temperature and vibration measurements.

Other studies have also been made to handle polarization fading in $\phi$-OTDR. This issue is introduced in measurements due to the fact that the state of polarization (SoP) of coherent Rayleigh backscattering signal is randomly distributed along the fiber owing to the random birefringence of the constituting material. This poses an issue in coherent detection schemes as the polarization of the local oscillator cannot be in alignment with that of the backscattered light. Resolving the problem with polarization maintaining fibers poses challenges due to the high loss and cost of such fibers. Recently, there has been theoretical investigation of this issue, and a method based on polarization diversity has been proposed and demonstrated [50]. It hinges on separating the backscattering signal into two orthogonal SoPs and a subsequent combination making sure that the detected signal is insensitive to the SoP along the fiber.

The experimental setup used to prove this scheme is depicted in Figure 17. As shown, first light from the external cavity laser (ECL) is divided in an 80:20 percentage ratio using an optical coupler. The $80 \%$ fraction is then fed to the AOM, which shifts the frequency by $200 \mathrm{MHz}$, and 


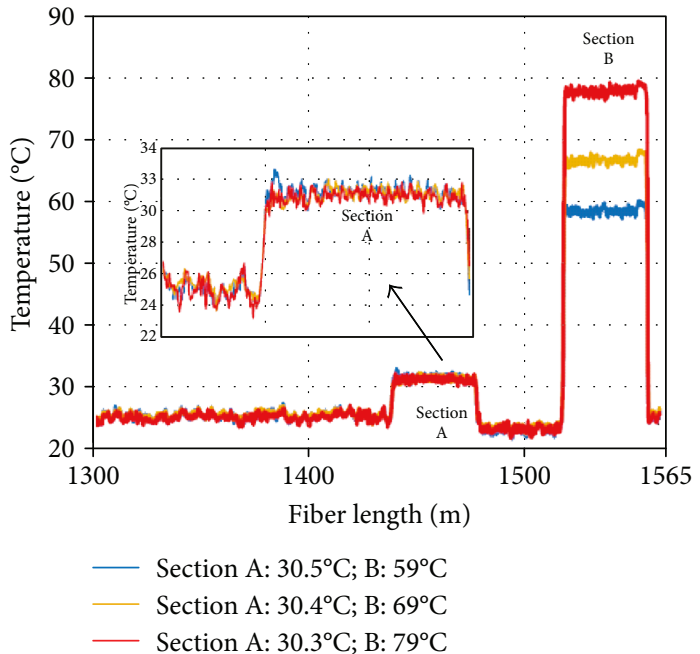

(a)

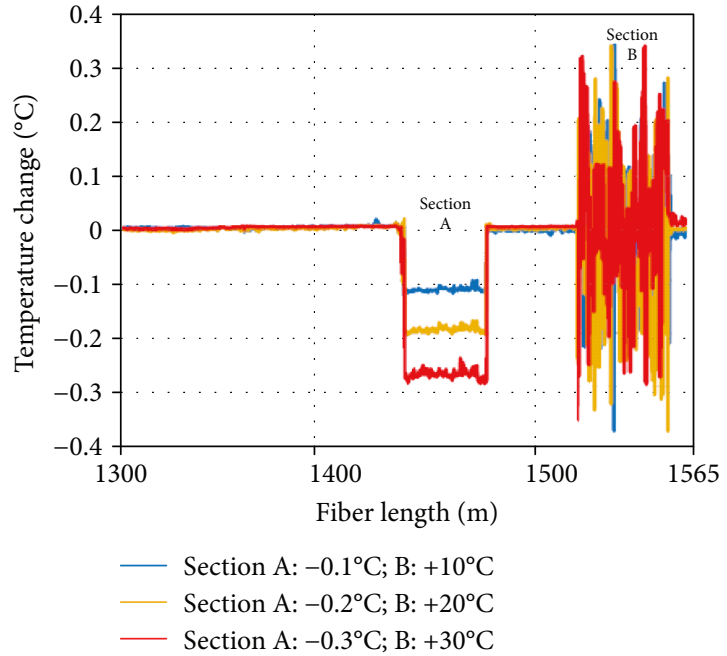

(b)

FIGURE 16: Distributed temperature profile near two sections A and B heated, respectively, with small and large temperature changes [49]: (a) BOTDA for profiling in section B and (b) $\phi$-OTDR for resolved profiling in section A.

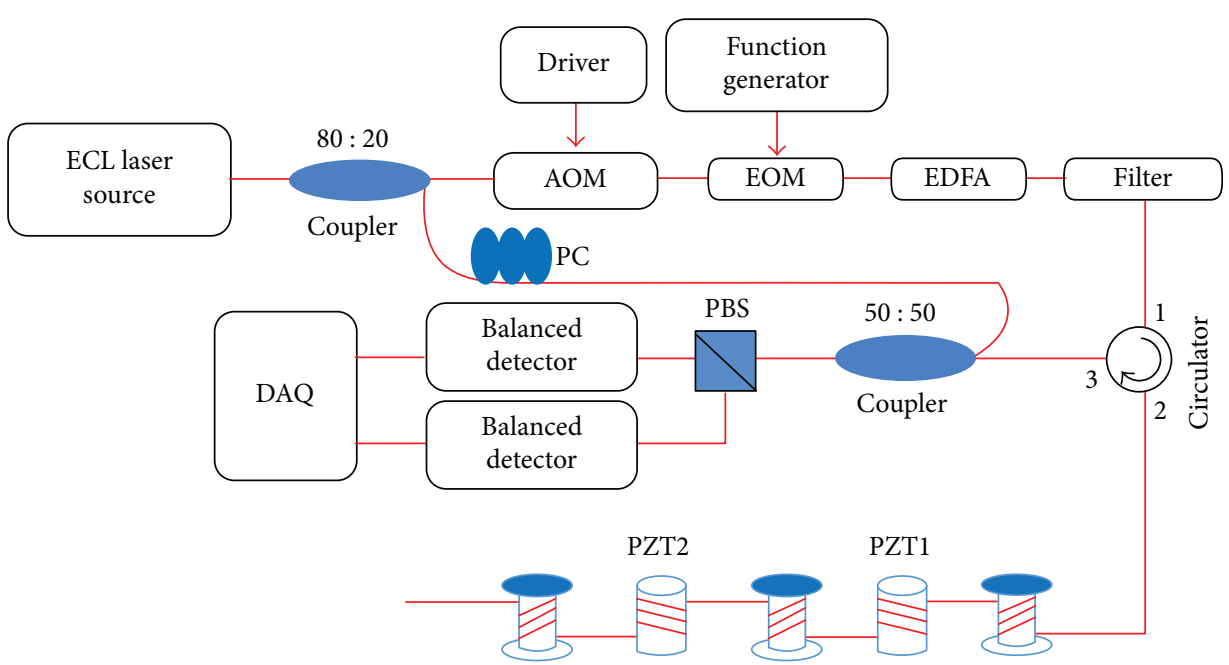

FIGURE 17: Experimental setup of using polarization diversity to address polarization fading in $\phi$-OTDR [50].

modulated with the EOM to generate pulses which are further amplified with the EDFA and filtered before being sent into the sensing fiber through the circulator. The backscattering from the fiber is then coupled with the $20 \%$ fraction of the source before being split with the polarization beam splitter (PBS) into two orthogonal SoPs. The postprocessing used involves summing the amplitudes of the squared signals from the two balanced photodetectors, to obtain a signal which is independent of the input SoP [50]. Using this technique, the SNR in measuring vibrations of $500 \mathrm{~Hz}$ has been improved by up to $10.9 \mathrm{~dB}$.

Finally, it is worth noting that, even though the scope of this review is limited to DAS techniques based on time domain, there are also implementations of DAS based on frequency domain techniques. These schemes use frequency modulated signals to probe the fiber, followed by the transformation of the response to its time domain equivalent to retrieve the spatial response. Since these techniques do not require the use of pulses, a typical OFDR offers spatially resolved measurements with high probing energy suitable for measurements in the presence of noise [51, 52]. Note also that there have recently been time-domain techniques which seek to address this long-standing issue of spatially resolved measurements, by decoupling the spatial resolution from the pulse width and demonstrating reduced measurement resolution down to few centimeters, albeit at a lower performance than frequency domain ones, as discussed in Section 5 [34].

Recent research on DAS based on OFDR has been focused, among others issues, on addressing the need for computationally-intensive algorithms for postprocessing of the acquired raw backscattering signal from the fiber to convert it to a suitable representation for extraction of sensing parameters, as it poses a challenge for real-time applications. 
Recently, one such study has sought to resolve this issue for a sinusoidal OFDR enabling the reduction of the complexity of the processing algorithm from $O\left(N^{2}\right)$ to $O(N \log N)$ to enable faster scan rates of $400 \mathrm{~Hz}$ at long distances [53].

Note also that additional schemes or signal processing methods such as phase demodulation techniques or others, which are discussed for DAS using time domain in Section 6 , have recently been studied in the frequency domain as well. They are required in order to perform more precise quantification of external parameters such as dynamic strain. Such implementations have been done in OFDR in [54] demonstrating spatially resolved measurements at $30 \mathrm{~m}$ with a sampling rate of $50 \mathrm{~Hz}$. More recently, a technique has been proposed to implement a phase demodulation scheme in OFDR for dynamic strain measurement which has already been demonstrated in $\phi$-OTDR [40]. The scheme is based on the use of a $3 \times 3$ optical coupler and enables quantitative measurement of a $100 \mathrm{~Hz}$ dynamic strain at $200 \mathrm{~m} \mathrm{[55].}$

\section{Summary}

To summarize, a review of the recent advances in DAS systems using $\phi$-OTDR has been presented. First, the operating principle and underlying physical mechanism used in $\phi$-OTDR for fast distributed measurements have been described. Then, some of the recent advances in performance enhancement in $\phi$-OTDR sensors for measuring backscattering intensity changes have been discussed. These include use of high ER pulses to reduce coherence noise, fast denoising in the optical domain using optical pulse coding technique, generation of high ER pulses using nonlinear Kerr effect, and the identification of pulse shapes robust against modulation instability. A technique to increase the intensity and SNR of the backscattering in a single-mode fiber using a cost-effective method not necessitating precise alignment required in writing FBGs, the use of a sample-and-hold analogue circuit which is capable of tuning vibration measurement to a particular point in the fiber, and a method which can be used to reconstruct sound signals applied on fiber through air in a similar manner to a microphone have also been presented. A parameter for the evaluation and comparison of the sensitivity performance of DAS systems has also been described. The treatment also includes that of new DAS configurations which advance the speed of measurement in $\phi$-OTDR beyond the previously established theoretical limit set by the sensing distance. These schemes are mainly based on the use of "colored" probe pulses or linear frequency multiplexing, which enable multiple sampling of perturbations at any spatial location at rates surpassing conventional limits set by the sensing distance.

In addition, an overview of some of the recent advances in improving the spatial resolution of $\phi$-OTDR sensors has been provided, including one based on tweaking of the conventional setup to reduce the optical noise when using short pulses with chirped pulse amplification techniques. Another notable technique performs decoupling of the width of the probing pulse from the spatial resolution by using linear frequency modulation at the source and a matched filter at the receiver, whose bandwidth determines the spatial resolution. Measurements with spatial resolution values down to few centimeters are reported using such advanced methods. Other recent schemes discussed in the review include those aimed at more precise quantitative measurement of an external impact using $\phi$-OTDR based on frequency shift measurements and direct phase demodulation methods. Frequency shift techniques include quasi-static frequency scanning of the probing pulse, chirping of a pulse for a single-shot measurement, and alternative frequency dual-pulse scheme involving correlation of the backscattering from the consecutive pulses in the pair. Phase demodulation techniques consist of digital coherent detection, a delayed interferometric scheme with a passive $3 \times 3$ coupler, a triplet of double pulses with relative phase shift emulating the $3 \times 3$ coupler, a double-pulse pair with relative frequency shift followed by I/Q mixing, and coherent homodyne detection with a $90^{\circ}$ hybrid. Finally, a sample hybrid technique which combines the complementary benefits of $\phi$-OTDR and Brillouin sensing, respectively, for a high resolution and high dynamic range measurement with a multicore fiber, and use of polarization diversity to address polarization fading in $\phi$-OTDR have been discussed. Overall, this contribution provides an overview of the recent advances and some areas of focus of research in $\phi$-OTDR configurations for DAS, which is a technology constituting a steadily increasing share of the fiber-optic sensor market, thanks to its many interesting applications in safety, security, and integrity monitoring systems.

\section{Conflicts of Interest}

The author declares that there is no conflict of interest regarding the publication of this paper.

\section{References}

[1] W. Lin, C. Zhang, L. Li, and S. Liang, "Review on development and applications of fiber-optic sensors," in 2012 Symposium on Photonics and Optoelectronics, pp. 1-4, Shanghai, China, 2012.

[2] A. Voskoboinik, A. Bozovich, A. E. Willner, and M. Tur, "Sweep-free Brillouin optical time-domain analyzer with extended dynamic range," in Conference on Lasers and Electro-Optics 2012, pp. 1-2, San Jose, CA, USA, 2012.

[3] A. Bergman, L. Yaron, T. Langer, and M. Tur, "Dynamic and distributed slope-assisted fiber strain sensing based on optical time-domain analysis of Brillouin dynamic gratings," Journal of Lightwave Technology, vol. 33, no. 12, pp. 2611-2616, 2015.

[4] K. Johannessen and B. K. Drakeley, "Distributed acoustic sensing - a new way of listening to your well/reservoir," in SPE Intelligent Energy International, Utrecht, Netherlands, 2012.

[5] Persistence Market Research, "Global distributed acoustic sensing market to surpass US\$ 2 billion in revenues by 2025," https://www.persistencemarketresearch.com/mediarelease/dis tributed-acoustic-sensing-market.asp.

[6] P. Healey, "Statistics of Rayleigh backscatter from a singlemode fiber," IEEE Transactions on Communications, vol. 35, no. 2, pp. 210-214, 1987.

[7] H. F. Martins, S. Martin-Lopez, P. Corredera, M. L. Filograno, O. Frazao, and M. Gonzalez-Herraez, "Coherent noise 
reduction in high visibility phase-sensitive optical time domain reflectometer for distributed sensing of ultrasonic waves," Journal of Lightwave Technology, vol. 31, no. 23, pp. 3631-3637, 2013.

[8] A. K. Wojcik, Signal Statistics of Phase Dependent Optical Time Domain Reflectometry, [Ph.D. Thesis], Department of Electrical Engineering and Computer Science, Texas A\&M University, College Station, TX, USA, 2006, http://oaktrust. library.tamu.edu/bitstream/handle/1969.1/4873/etd-tamu2006C-ELEN-Wojcik.pdf? sequence=1.

[9] Y. Shi, H. Feng, and Z. Zeng, "A long distance phase-sensitive optical time domain reflectometer with simple structure and high locating accuracy," Sensors, vol. 15, no. 9, pp. 2195721970, 2015.

[10] J. Park and H. F. Taylor, "Fiber optic intrusion sensor using coherent optical time domain reflectometer," Japanese Journal of Applied Physics, vol. 42, 6A, Part 1, pp. 3481-3482, 2003.

[11] J. C. Juarez, E. W. Maier, K. N. Choi, and H. F. Taylor, "Distributed fiber-optic intrusion sensor system," Journal of Lightwave Technology, vol. 23, no. 6, pp. 2081-2087, 2005.

[12] Y. Lu, T. Zhu, L. Chen, and X. Bao, "Distributed vibration sensor based on coherent detection of phase-OTDR," Journal of Lightwave Technology, vol. 28, no. 22, pp. 3243-3249, 2010.

[13] Z. Qin, T. Zhu, L. Chen, and X. Bao, "High sensitivity distributed vibration sensor based on polarization-maintaining configurations of phase-OTDR," IEEE Photonics Technology Letters, vol. 23, no. 15, pp. 1091-1093, 2011.

[14] Z. Qin, L. Chen, and X. Bao, "Wavelet denoising method for improving detection performance of distributed vibration sensor," IEEE Photonics Technology Letters, vol. 24, no. 7, pp. 542544, 2012.

[15] H. F. Martins, S. Martin-Lopez, P. Corredera, P. Salgado, O. Frazão, and M. González-Herráez, "Modulation instability-induced fading in phase-sensitive optical timedomain reflectometry," Optics Letters, vol. 38, no. 6, pp. 872874, 2013.

[16] Y. Muanenda, C. J. Oton, S. Faralli, and F. Di Pasquale, "High performance distributed acoustic sensor using cyclic pulse coding in a direct detection coherent-OTDR," in Fifth AsiaPacific Optical Sensors Conference, p. 965547, Jeju, Republic of Korea, 2015.

[17] Y. Muanenda, C. Oton, T. Nannipieri, A. Signorini, S. Faralli, and F. Di Pasquale, "Hybrid distributed optical fiber sensors for temperature, strain and vibration measurements," in 2015 International Conference on Microwave, Optical and Communication Engineering (ICMOCE), pp. 1-4, Bhubaneswar, India, 2015.

[18] Y. Muanenda, C. J. Oton, S. Faralli, T. Nannipieri, A. Signorini, and F. Di Pasquale, "A distributed acoustic and temperature sensor using a commercial off-the-shelf DFB laser," in 24th International Conference on Optical Fibre Sensors, p. 96342C, Curitiba, PR, Brazil, 2015.

[19] Y. Muanenda, C. J. Oton, S. Faralli, T. Nannipieri, A. Signorini, and F. D. Pasquale, "Hybrid distributed acoustic and temperature sensor using a commercial off-the-shelf DFB laser and direct detection," Optics Letters, vol. 41, no. 3, pp. 587-590, 2016.

[20] C. Baker, B. Vanus, M. Wuilpart, L. Chen, and X. Bao, "Enhancement of optical pulse extinction-ratio using the nonlinear Kerr effect for phase-OTDR," Optics Express, vol. 24, no. 17, pp. 19424-19434, 2016.
[21] C. Franciscangelis, W. Margulis, L. Kjellberg, I. Soderquist, and F. Fruett, "Real-time distributed fiber microphone based on phase-OTDR," Optics Express, vol. 24, no. 26, pp. $29597-$ 29602, 2016.

[22] S. Loranger, M. Gagné, V. Lambin-Iezzi, and R. Kashyap, "Rayleigh scatter based order of magnitude increase in distributed temperature and strain sensing by simple UV exposure of optical fibre," Scientific Reports, vol. 5, no. 1, article 11177, 2015.

[23] M. R. Fernández-Ruiz, H. F. Martins, J. Pastor-Graells, S. Martin-Lopez, and M. Gonzalez-Herraez, "Phase-sensitive OTDR probe pulse shapes robust against modulationinstability fading," Optics Letters, vol. 41 , no. 24 , pp. $5756-$ 5759, 2016.

[24] Z. Qin, H. Chen, and J. Chang, "Signal-to-noise ratio enhancement based on empirical mode decomposition in phasesensitive optical time domain reflectometry systems," Sensors, vol. 17, no. 8, 2017.

[25] P. Golacki, A. Masoudi, K. Holland, and T. Newson, "Distributed optical fibre acoustic sensors - future applications in audio and acoustics engineering," in Proceedings of the Institute of Acoustics, vol. 38, Part 2, Kenilworth, UK, 2016.

[26] L. S. Christensen and D. Manvell, "Sound level meters in building acoustic measurements," Building Acoustics, vol. 5, no. 3, pp. 217-222, 1998.

[27] F. Bettarello, P. Fausti, V. Baccan, and M. Caniato, "Impact sound pressure level performances of basic beam floor structures," Building Acoustics, vol. 17, no. 4, pp. 305-316, 2010.

[28] H. Gabai and A. Eyal, "How to specify and measure sensitivity in distributed acoustic sensing (DAS)?," in 25th International Conference on Optical Fiber Sensors, pp. 1-4, Jeju Island, Republic of Korea, 2017.

[29] D. Iida, K. Toge, and T. Manabe, "High-frequency distributed acoustic sensing faster than repetition limit with frequencymultiplexed phase-OTDR," in 2016 Optical Fiber Communications Conference and Exhibition (OFC), pp. 1-3, Anaheim, CA, USA, 2016.

[30] D. Chen, Q. Liu, X. Fan, and Z. He, "Distributed fiber-optic acoustic sensor with enhanced response bandwidth and high signal-to-noise ratio," Journal of Lightwave Technology, vol. 35, no. 10, pp. 2037-2043, 2017.

[31] Q. Liu, X. Fan, and Z. He, "Time-gated digital optical frequency domain reflectometry with $1.6-\mathrm{m}$ spatial resolution over entire 110-km range," Optics Express, vol. 23, no. 20, pp. 25988-25995, 2015.

[32] S. Wang, X. Fan, Q. Liu, and Z. He, "Distributed fiber-optic vibration sensing based on phase extraction from time-gated digital OFDR," Optics Express, vol. 23, no. 26, pp. 3330133309, 2015.

[33] J. Pastor-Graells, L. R. Cortés, M. R. Fernández-Ruiz et al., "SNR enhancement in high-resolution phase-sensitive OTDR systems using chirped pulse amplification concepts," Optics Letters, vol. 42, no. 9, pp. 1728-1731, 2017.

[34] B. Lu, Z. Pan, Z. Wang et al., "High spatial resolution phasesensitive optical time domain reflectometer with a frequencyswept pulse," Optics Letters, vol. 42, no. 3, pp. 391-394, 2017.

[35] Z. Sha, H. Feng, and Z. Zeng, "Phase demodulation method in phase-sensitive OTDR without coherent detection," Optics Express, vol. 25, no. 5, pp. 4831-4844, 2017.

[36] Y. Koyamada, M. Imahama, K. Kubota, and K. Hogari, "Fiberoptic distributed strain and temperature sensing with very 
high measurand resolution over long range using coherent OTDR," Journal of Lightwave Technology, vol. 27, no. 9, pp. 1142-1146, 2009.

[37] A. Garcia-Ruiz, J. Pastor-Graells, H. F. Martins, S. MartinLopez, and M. Gonzalez-Herraez, "Speckle analysis method for distributed detection of temperature gradients with $\Phi$ OTDR," IEEE Photonics Technology Letters, vol. 28, no. 18, pp. 2000-2003, 2016.

[38] J. Pastor-Graells, H. F. Martins, A. Garcia-Ruiz, S. MartinLopez, and M. Gonzalez-Herraez, "Single-shot distributed temperature and strain tracking using direct detection phasesensitive OTDR with chirped pulses," Optics Express, vol. 24, no. 12, pp. 13121-13133, 2016.

[39] S. Liehr, Y. S. Muanenda, S. Münzenberger, and K. Krebber, "Relative change measurement of physical quantities using dual-wavelength coherent OTDR," Optics Express, vol. 25, no. 2, pp. 720-729, 2017.

[40] A. Masoudi, M. Belal, and T. P. Newson, “A distributed optical fibre dynamic strain sensor based on phase-OTDR," Measurement Science and Technology, vol. 24, no. 8, article 085204, 2013.

[41] A. Masoudi and T. P. Newson, "High spatial resolution distributed optical fiber dynamic strain sensor with enhanced frequency and strain resolution," Optics Letters, vol. 42, no. 2, pp. 290-293, 2017.

[42] A. E. Alekseev, V. S. Vdovenko, B. G. Gorshkov, V. T. Potapov, and D. E. Simikin, "A phase-sensitive optical time-domain reflectometer with dual-pulse phase modulated probe signal," Laser Physics, vol. 24, no. 11, article 115106, 2014.

[43] A. E. Alekseev, V. S. Vdovenko, B. G. Gorshkov, V. T. Potapov, and D. E. Simikin, "A phase-sensitive optical time-domain reflectometer with dual-pulse diverse frequency probe signal," Laser Physics, vol. 25, no. 6, article 065101, 2015.

[44] Z. Wang, L. Zhang, S. Wang et al., "Coherent $\Phi$-OTDR based on I/Q demodulation and homodyne detection," Optics Express, vol. 24, no. 2, pp. 853-858, 2016.

[45] Z. Pan, K. Liang, Q. Ye, H. Cai, R. Qu, and Z. Fang, "Phasesensitive OTDR system based on digital coherent detection," in Optical Sensors and Biophotonics, p. 83110S, Shanghai, China, 2011.

[46] J. C. Estrada, M. Servin, and J. Vargas, "2D Simultaneous phase unwrapping and filtering: a review and comparison," Optics and Lasers in Engineering, vol. 50, no. 8, pp. 1026-1029, 2012.

[47] D. Kitahara, M. Yamagishi, and I. Yamada, "A virtual resampling technique for algebraic two-dimensional phase unwrapping," in 2015 IEEE International Conference on Acoustics, Speech and Signal Processing (ICASSP), pp. 3871-3875, Brisbane, QLD, Australia, 2015.

[48] N. H. Ching, D. Rosenfeld, and M. Braun, "Two-dimensional phase unwrapping using a minimum spanning tree algorithm," IEEE Transactions on Image Processing, vol. 1, no. 3, pp. 355-365, 1992.

[49] Y. Dang, Z. Zhao, M. Tang et al., "Towards large dynamic range and ultrahigh measurement resolution in distributed fiber sensing based on multicore fiber," Optics Express, vol. 25, no. 17, article 20183, 20193 pages, 2017.

[50] M. Ren, P. Lu, L. Chen, and X. Bao, “Theoretical and experimental analysis of $\Phi$-OTDR based on polarization diversity detection," IEEE Photonics Technology Letters, vol. 28, no. 6, pp. 697-700, 2016.
[51] H. Gabai and A. Eyal, "Distributed audio recording using OFDR with double interrogation," in 23rd International Conference on Optical Fibre Sensors, p. 91576C, Santander, Spain, 2014.

[52] H. Gabai, Y. Botsev, M. Hahami, and A. Eyal, "Optical frequency domain reflectometry at maximum update rate using I/Q detection," Optics Letters, vol. 40, no. 8, pp. 1725-1728, 2015.

[53] L. Shiloh and A. Eyal, "Sinusoidal frequency scan OFDR with fast processing algorithm for distributed acoustic sensing," Optics Express, vol. 25, no. 16, pp. 19205-19215, 2017.

[54] D.-P. Zhou, L. Chen, and X. Bao, "Distributed dynamic strain measurement using optical frequency-domain reflectometry," Applied Optics, vol. 55, no. 24, pp. 6735-6739, 2016.

[55] J. Li, J. Gan, Z. Zhang et al., "High spatial resolution distributed fiber strain sensor based on phase-OFDR," Optics Express, vol. 25, no. 22, pp. 27913-27922, 2017. 


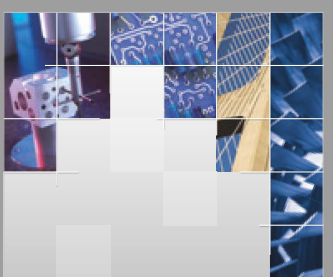

\section{Enfincering}
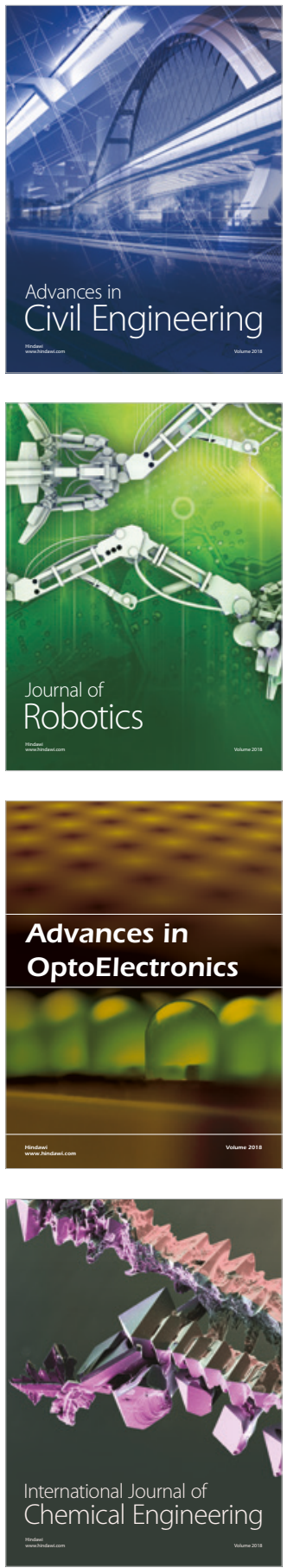

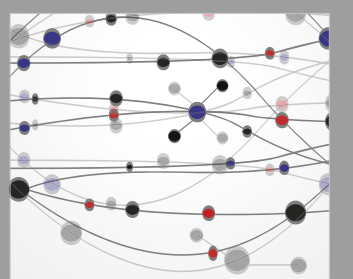

\section{Rotating \\ Machinery}

The Scientific World Journal

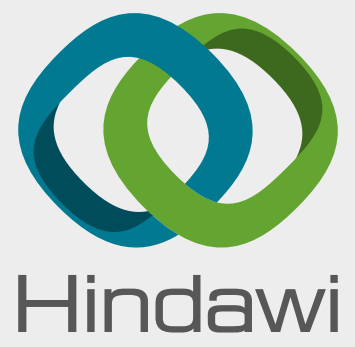

Submit your manuscripts at

www.hindawi.com
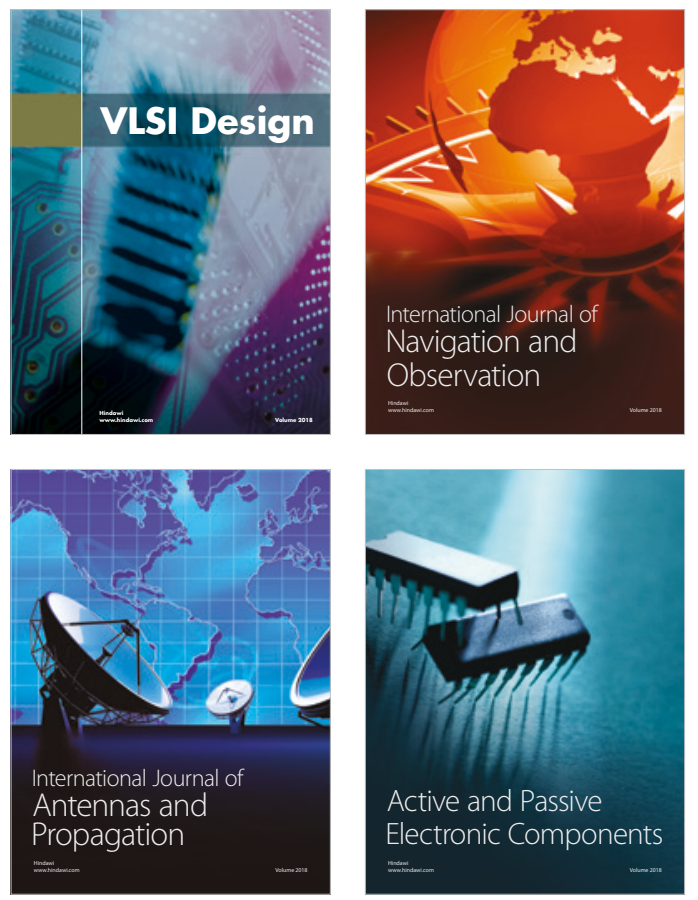
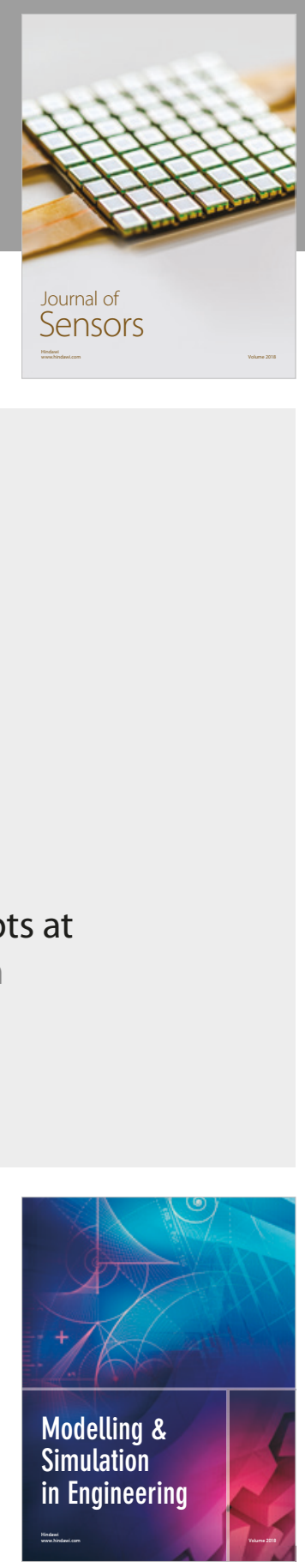

\section{Advances \\ Multimedia}
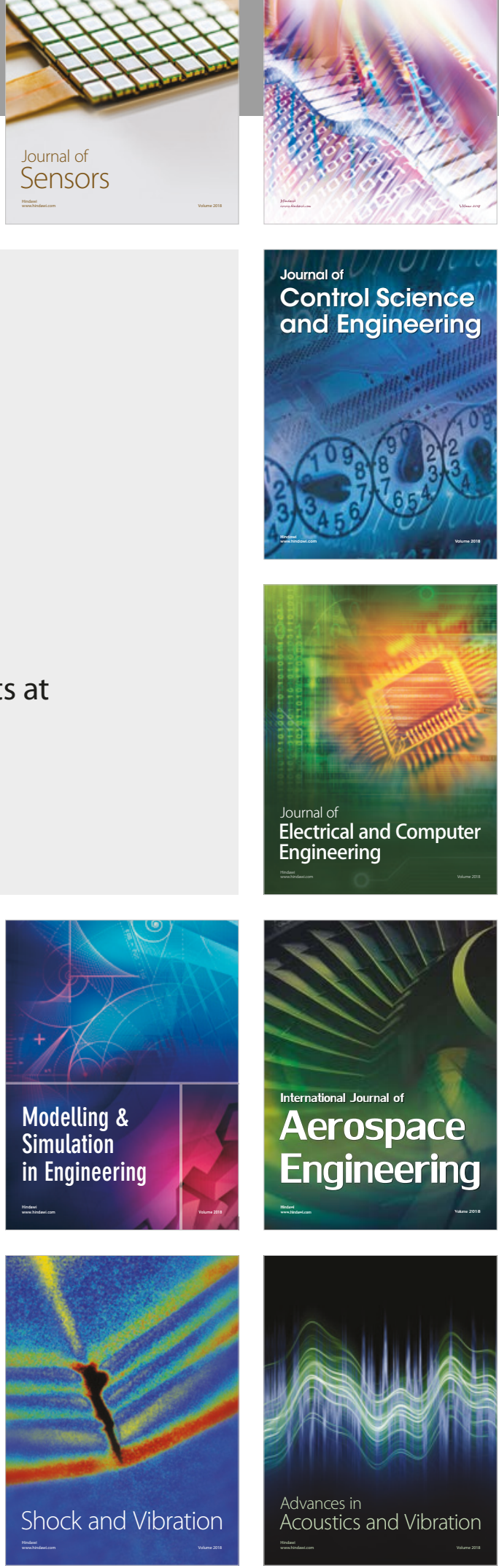